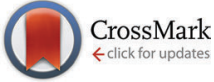

Cite this: Phys. Chem. Chem. Phys., 2016, 18, 28242

Received 15th August 2016, Accepted 26th September 2016

DOI: 10.1039/c6cp05653a

www.rsc.org/pccp

\section{Liquid silver tris(perfluoroethyl)trifluorophosphate salts as new media for propene/propane separation $\dagger$}

\author{
Daniel Pliquett, ${ }^{a}$ Peter S. Schulz, ${ }^{a}$ Frank W. Heinemann, ${ }^{b}$ Angela Bause ${ }^{c}$ and \\ Peter Wasserscheid*a
}

\begin{abstract}
A series of silver tris(perfluoroethyl)trifluorophosphate (Ag[FAP]) complexes with various ligands (acetonitrile ACN, chloroacetonitrile $\mathrm{Cl}-\mathrm{ACN}$, acrylonitrile acryl- $\mathrm{CN}$, pyridine py, ethylenediamine en and propene $\mathrm{C}_{3} \mathrm{H}_{6}$ )

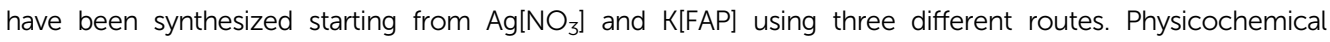
properties as well as crystal structures ([Ag(ACN) $\left.\left.)_{2 / 4}\right][\mathrm{FAP}],\left[\mathrm{Ag}(\mathrm{py})_{2}\right][\mathrm{FAP}]\right)$ were determined and the suitability of such Ag salts for propene/propane separation processes was investigated. The investigated silver complexes exhibit either low melting points or form liquid complexes when contacted with gaseous propene at $30{ }^{\circ} \mathrm{C}$. This makes them promising separation materials for both liquid membranes and absorber fluids due to their high silver content and significant propene capacity. Single (iGSC) and mixed (NMR) gas solubilities as well as diffusion coefficients (PFG-NMR) of propene and propane were determined to predict the theoretical selectivity of solubility, membrane selectivity, capacity and transport properties of the silver salts according to the solution diffusion model. A strong influence of the number and type of ligands on chemical complexation, physicochemical properties and separation performance has been observed.
\end{abstract}

\section{Introduction}

Recent studies state a worldwide propene consumption of 85 million tons for 2013 and predict a continuous growth of propylene production of $\sim 5 \%$ p.a. until 2021 . $^{1}$ These numbers clearly demonstrate the current and future importance of propene for the petrochemical industry. Today, propene is mainly produced in steam crackers, FCC units or in on-purpose reactions like propane dehydrogenation. All these production routes are characterized by the fact that propene is obtained in crude or preseparated product mixtures that contain significant amounts of propane. The current standard separation process to isolate propene from these mixtures in high purity is cryogenic distillation, however, this process suffers from its very high capital costs and an enormous energy consumption making up for approximately $75 \%$ of the total olefin production costs according to Kim et $a .^{2}$ With the increasing

\footnotetext{
${ }^{a}$ Friedrich-Alexander-University Erlangen-Nürnberg (FAU), Institute of Chemical Reaction Engineering, Egerlandstraße 3, 91058 Erlangen, Germany. E-mail: peter.wasserscheid@fau.de; Fax: +499131/8527421; Tel: +499131/8527420

${ }^{b}$ Friedrich-Alexander-University Erlangen-Nürnberg (FAU), Chair of Inorganic and General Chemistry, Egerlandstraße 1, 91058 Erlangen, Germany

${ }^{c}$ Merck KGaA, Frankfurter Str. 250, 64293 Darmstadt, Germany

$\dagger$ CCDC 1498195-1498197. Crystallographic data, density and thermal stability data, NMR and ESI-MS spectra of [Ag(ACN $\left.)_{2}\right][\mathrm{FAP}]$, solubility, capacity, diffusion and selectivity data of the silver salts. For crystallographic data in CIF or other electronic format see DOI: 10.1039/c6cp05653a
}

demand for propylene and the expansion and new construction of production sites over the last years, the need for new propene/ propane separation processes and the chance for new technologies to be applied is rising. ${ }^{3}$ Thus, a variety of different approaches and processes have been proposed to enable more efficient propene/ propane separation. ${ }^{4,5}$ Among the proposed concepts, separation by chemical complexation mechanisms in membrane or by absorption/ desorption processes appear as most promising.

The addition of silver salts to water or ionic liquids has been shown in the past to drastically enhance both, alkene capacity and selectivity due to the known chemical interaction of the alkene with the silver ion. ${ }^{6-14}$ Aqueous silver nitrate and silver tetrafluoroborate solutions have been extensively studied and were close to commercialization in absorption/desorption processes. ${ }^{10,15,16}$ Yet, both silver salts show tendencies to form explosive silver acetylides or decompose in aqueous environments. Furthermore, evaporation of water in the desorption process can not be avoided. These disadvantages are overcome by using ionic liquids as solvents, as these liquid salts are characterized by very low vapor pressures. Consequently, solutions of $\mathrm{Ag}\left[\mathrm{NTf}_{2}\right], \mathrm{Ag}\left[\mathrm{NO}_{3}\right]$, $\mathrm{Ag}\left[\mathrm{BF}_{4}\right]$ and $\mathrm{Ag}[\mathrm{OTf}]$ in various ionic liquids have also been investigated with respect to their olefin uptake and their application in ene/ane separation processes has been suggested. ${ }^{11-14}$ Dissolution of solid silver salts in an ionic liquid, however, always comes with a decrease in silver concentration and thus decreasing capacity for alkene complexation. Furthermore, an 
increase in viscosity with increasing silver concentration is generally observed, leading to slower mass transport. ${ }^{17}$ Therefore, it seems desirable to employ neat liquid silver salts for ene/ane separation for the following reasons:

- high capacity

- no solvent evaporation

- high flux when applied in form of liquid membranes.

Low melting silver salts have been studied by the group of Binnemans for electrodeposition purposes. ${ }^{18-22}$ It has been shown that by adding different ligands to the silver center, the melting point of usually high melting silver salts can be significantly reduced by disturbing the crystal packing. Depending on the number and structure of the ligand in combination with a suitable anion, melting points below $100{ }^{\circ} \mathrm{C}$ could be observed for example for $\left[\mathrm{Ag}(\text { hexylimidazolyl })_{2}\right]\left[\mathrm{NO}_{3}\right](\mathrm{mp}<\mathrm{rt}){ }^{19}$ $\left[\mathrm{Ag}(\right.$ methylimidazolyl)(butylimidazolyl) $]\left[\mathrm{NTf}_{2}\right]\left(\mathrm{mp} 30^{\circ} \mathrm{C}\right)^{18}$ or $\left.[\text { Ag(pyridine- } N \text {-oxide })_{3}\right][$ OTf $]\left(\operatorname{mp} 76{ }^{\circ} \mathrm{C}\right) .{ }^{21}$ Similar liquid silver salt complexes of $\mathrm{Ag}\left[\mathrm{NTf}_{2}\right]$ with dimethylbenzylamine, 1-propylamine, 1-pentene, 1-hexene, 1-heptene, 1-octene and isoprene were described by Huang et $a .^{23}$ and Wang et al. ${ }^{24,25}$ While Wang tested the silver ionic liquids for liquid phase separation of C5-C8 alkenes from ene/ane mixtures, Huang employed them in a liquid membrane for pentene/pentane and hexene/ hexane separation.

First examples of liquid silver salt complexes formed with gaseous alkene ligands were described by Francis, who discovered that $\mathrm{Ag}\left[\mathrm{NO}_{3}\right]$ forms a liquid phase with propene and 1-butene at low temperatures and pressures near the vapor pressure of the respective alkene. ${ }^{26}$ In contrast, $\mathrm{Ag}\left[\mathrm{BF}_{4}\right], \mathrm{Ag}\left[\mathrm{SbF}_{6}\right]$ and $\mathrm{Ag}\left[\mathrm{ClO}_{4}\right]$ are known to incorporate propene in their crystal lattice, forming solid complexes. ${ }^{27,28}$

To our best knowledge, the first example of a liquid silver propene complex at low pressures has been $\left[\operatorname{Ag}(\text { propene })_{x}\right]\left[\mathrm{NTf}_{2}\right]$, published by our group in $2011 .^{29,30}$ While [Ag(ethene $\left.)_{2}\right]\left[\mathrm{NTf}_{2}\right]$ is still a solid at room temperature, ${ }^{31}\left[\mathrm{Ag}(\text { propene })_{x}\right]\left[\mathrm{NTf}_{2}\right]$ solidifies only below $0{ }^{\circ} \mathrm{C}$ and exhibits low viscosity.

The availability of new anions allows for the preparation of new silver salts with potentially interesting properties for ene/ane separation. In this context, metal salts of the tris(perfluoroethyl)trifluorophosphate $\left([\mathrm{FAP}]^{-}\right.$) anion have caught our attention. $[\mathrm{FAP}]^{-}$-based ionic liquids have been introduced by Merck KGaA in $2005^{32,33}$ and are characterized by a number of very interesting properties such as very low nucleophilicity and very high hydrophobicity combined with good hydrolytic stability. ${ }^{34,35}$ Known examples of $\mathrm{Ag}[\mathrm{FAP}]$ complexes include $\left[\mathrm{Ag}(\mathrm{ACN})_{4}\right][\mathrm{FAP}]$ and $\left[\mathrm{Ag}(\text { toluene })_{2}\right][\mathrm{FAP}] .^{36}$

In this paper we report new synthetic pathways for $\mathrm{Ag}[\mathrm{FAP}]$ complexes with various ligands (acetonitrile ACN, chloroacetonitrile Cl-ACN, acrylonitrile acryl-CN, pyridine py, ethylenediamine en, propene $\mathrm{C}_{3} \mathrm{H}_{6}$ ). Moreover, the formation and physicochemical properties of their respective liquid propene complexes are presented. From the obtained data conclusions regarding their applicability for propene/propane separation processes, such as supported liquid membranes or absorption/desorption processes, are made. Separation selectivities and transport properties are predicted based on the solution diffusion model for liquid membranes and from the determined equilibrium absorption selectivities.

\section{Experimental}

\section{Materials}

K[FAP] was provided by Merck and ethylenediamine (99\%) was purchased from Alfa Aesar. Propene (2.5) and propane (2.5) were obtained from Linde. All other chemicals were purchased from Sigma-Aldrich with a purity of $99 \%$ or higher. All commercial materials were used as received.

\section{Synthetic procedures for the preparation of propene free silver salts}

General procedure for the synthesis of $\left[\mathrm{Ag}(\mathrm{ACN})_{4}\right][\mathrm{FAP}]$, $\left[\mathrm{Ag}(\mathrm{Cl}-\mathrm{ACN})_{2}\right][\mathrm{FAP}]\left[\mathrm{Ag}(\operatorname{acryl}-\mathrm{CN})_{2}\right][\mathrm{FAP}]$ and $\left[\mathrm{Ag}(\mathbf{p y})_{2}\right][\mathrm{FAP}]$. At $0{ }^{\circ} \mathrm{C}$ a solution of K[FAP] in the respective solvent was slowly added to a solution of $\mathrm{Ag}\left[\mathrm{NO}_{3}\right]$ in the respective solvent under argon atmosphere and light exclusion. The reaction mixture was allowed to warm to room temperature and stirred for at least $23 \mathrm{~h}$. The precipitated $\mathrm{K}\left[\mathrm{NO}_{3}\right]$ was filtered off and washed with the respective solvent. After concentration at the rotary evaporator, the combined product phases were purified by washing with cold water and $n$-pentane. Drying at reduced pressure and temperatures of 25-50 ${ }^{\circ} \mathrm{C}$ resulted in the formation of the silver salt complexes with the stated compositions. The drying progress was monitored by NMR measurements.

Tetrakis(acetonitrile)silver(I)-tris(perfluoroethyl)trifluorophosphate $\left[\mathbf{A g}(\mathbf{A C N})_{4}\right][\mathbf{F A P}]$. The reaction of $35.09 \mathrm{~g}(206.56 \mathrm{mmol})$ $\mathrm{Ag}\left[\mathrm{NO}_{3}\right]$ in $100 \mathrm{~mL}$ acetonitrile with $100.00 \mathrm{~g}$ (206.56 mmol) $\mathrm{K}[\mathrm{FAP}]$ in $230 \mathrm{~mL}$ acetonitrile yielded $139.33 \mathrm{~g}(194.30 \mathrm{mmol}$, $94.07 \%)\left[\mathrm{Ag}(\mathrm{ACN})_{4}\right][\mathrm{FAP}]$ as colorless crystals. In contrast to the other complexes described below, slow and careful drying is necessary to form $\left[\mathrm{Ag}(\mathrm{ACN})_{4}\right][\mathrm{FAP}]$. mp: $44{ }^{\circ} \mathrm{C} .{ }^{1} \mathrm{H}$ NMR $(400 \mathrm{MHz}$, $\mathrm{d}_{4}$-MeOD): $\delta / \mathrm{ppm}=2.10\left(\mathrm{~s}, 12 \mathrm{H}, \mathrm{CH}_{3}\right) \cdot{ }^{13} \mathrm{C} \mathrm{NMR}(101 \mathrm{MHz}$, $\mathrm{d}_{4}$-MeOD): $\delta / \mathrm{ppm}=0.82\left(\mathrm{q},{ }^{1} J_{\mathrm{C}, \mathrm{H}}=5.72 \mathrm{~Hz}, 4 \mathrm{C}, \mathrm{CH}_{3}\right), 119.33$ (s, 4C, CN), $121.52\left(\mathrm{~m}, 6 \mathrm{C}, 3 \mathrm{C}_{2} \mathrm{~F}_{5}\right) .{ }^{19} \mathrm{~F} \mathrm{NMR}$ (376 MHz, d $\left.\mathrm{d}_{4}-\mathrm{MeOD}\right)$ : $\delta / \mathrm{ppm}=-117.27\left(\mathrm{md},{ }^{2} J_{\mathrm{F}, \mathrm{P}}=97.42 \mathrm{~Hz}, 4 \mathrm{~F}, 2 \mathrm{CF}_{2}\right.$ ), -116.67 (br d, $\left.{ }^{2} J_{\mathrm{F}, \mathrm{P}}=83.13 \mathrm{~Hz}, 2 \mathrm{~F}, 1 \mathrm{CF}_{2}\right),-88.78\left(\mathrm{md},{ }^{1} J_{\mathrm{F}, \mathrm{P}}=901.98 \mathrm{~Hz}, 2 \mathrm{~F}, \mathrm{PF}_{2}\right)$, $-83.04\left(\mathrm{~m}, 6 \mathrm{~F}, 2 \mathrm{CF}_{3}\right),-81.34\left(\mathrm{~m}, 3 \mathrm{~F}, 1 \mathrm{CF}_{3}\right),-45.46\left(\mathrm{md},{ }^{1} J_{\mathrm{F}, \mathrm{P}}=\right.$ $889.57 \mathrm{~Hz}, 1 \mathrm{~F}, \mathrm{PF}) .{ }^{31} \mathrm{P}$ NMR (162 MHz, d $\left.\mathrm{d}_{4}-\mathrm{MeOD}\right): \delta / \mathrm{ppm}=$ $-147.26\left(\mathrm{mtd},{ }^{1} J_{\mathrm{P}, \mathrm{F}}={ }^{1} J_{\mathrm{P}, \mathrm{F}}=899.53 \mathrm{~Hz}, 1 \mathrm{P}\right) .{ }^{1} \mathrm{H}$ qNMR $(400 \mathrm{MHz}$, $\mathrm{d}_{4}$-MeOD, 1,3-bis(trifluoromethyl)benzene): $n($ ACN) $=3.99$. ESI-MS $\left(\mathrm{H}_{2} \mathrm{O}\right.$, negative mode): $m / z=445.0$ (100\%), 446.1 (9). ESI-MS $\left(\mathrm{H}_{2} \mathrm{O}\right.$, positive mode): $m / z=106.9$ (54\%), 108.9 (42), 124.9 (22), 126.9 (19), 143.1 (11), 144.9 (10), 147.9 (100), 149.9 (87), 166.0 (9), 167.9 (7), 189.0 (22), 190.8 (19), 205.9 (8), 207.8 (8). ICP-AES $\left(\mathrm{H}_{2} \mathrm{O}\right)$ : $n(\mathrm{Ag}) / n(\mathrm{P})=1.02$.

Bis(acetonitrile)silver(I)-tris(perfluoroethyl)trifluorophosphate $\left[\mathbf{A g}(\mathbf{A C N})_{2}\right][\mathbf{F A P}] \cdot\left[\mathrm{Ag}(\mathrm{ACN})_{2}\right][\mathrm{FAP}]$ was obtained quantitatively as colorless crystals by treating $\left[\mathrm{Ag}(\mathrm{ACN})_{4}\right][\mathrm{FAP}]$ for three days under high vacuum at moderate temperatures (max. $\left.50{ }^{\circ} \mathrm{C}\right) . \mathrm{mp}: 31{ }^{\circ} \mathrm{C}$. ${ }^{1} \mathrm{H}$ NMR (400 MHz, d $\left.\mathrm{d}_{4} \mathrm{MeOD}\right): \delta / \mathrm{ppm}=2.11\left(\mathrm{~s}, 6 \mathrm{H}, \mathrm{CH}_{3}\right)$. ${ }^{13} \mathrm{C}$ NMR (101 MHz, d $\left.\mathrm{d}_{4}-\mathrm{MeOD}\right): \delta / \mathrm{ppm}=0.78\left(\mathrm{q},{ }^{1} J_{\mathrm{C}, \mathrm{H}}=5.73 \mathrm{~Hz}\right.$, 
2C, $\mathrm{CH}_{3}$ ), $119.76(\mathrm{~s}, 2 \mathrm{C}, \mathrm{CN}), 121.47\left(\mathrm{~m}, 6 \mathrm{C}, 3 \mathrm{C}_{2} \mathrm{~F}_{5}\right) .{ }^{19} \mathrm{~F}$ NMR (376 MHz, d $\left.\mathrm{d}_{4}-\mathrm{MeOD}\right): \delta / \mathrm{ppm}=-117.49\left(\mathrm{md},{ }^{2} J_{\mathrm{F}, \mathrm{P}}=97.42 \mathrm{~Hz}, 4 \mathrm{~F}\right.$, $\left.2 \mathrm{CF}_{2}\right),-116.87\left(\mathrm{md},{ }^{2} J_{\mathrm{F}, \mathrm{P}}=83.13 \mathrm{~Hz}, 2 \mathrm{~F}, 1 \mathrm{CF}_{2}\right),-89.01\left(\mathrm{md},{ }^{1} J_{\mathrm{F}, \mathrm{P}}=\right.$ 902.36 Hz, 2F, $\left.\mathrm{PF}_{2}\right),-83.19\left(\mathrm{~m}, 6 \mathrm{~F}, 2 \mathrm{CF}_{3}\right),-81.49\left(\mathrm{~m}, 3 \mathrm{~F}, 1 \mathrm{CF}_{3}\right)$, $-45.80\left(\mathrm{md},{ }^{1} J_{\mathrm{F}, \mathrm{P}}=889.95 \mathrm{~Hz}, 1 \mathrm{~F}, \mathrm{PF}\right) .{ }^{31} \mathrm{P} \mathrm{NMR}(162 \mathrm{MHz}$, $\left.\mathrm{d}_{4}-\mathrm{MeOD}\right): \delta / \mathrm{ppm}=-147.27\left(\mathrm{mtd},{ }^{1} J_{\mathrm{P}, \mathrm{F}}={ }^{1} J_{\mathrm{P}, \mathrm{F}}=899.53 \mathrm{~Hz}, 1 \mathrm{P}\right)$. ${ }^{1} \mathrm{H}$ qNMR (400 MHz, d 4 -MeOD, 1,3-bis(trifluoromethyl)benzene): $n(\mathrm{ACN})=2.03$. ESI-MS $\left(\mathrm{H}_{2} \mathrm{O}\right.$, negative mode $): m / z=118.9(67 \%)$, 207.0 (12), 345.0 (35), 443.8 (5), 444.7 (100), 446.2 (38). ESI-MS $\left(\mathrm{H}_{2} \mathrm{O}\right.$, positive mode): $\mathrm{m} / \mathrm{z}=106.9$ (100\%), 108.8 (99), 125.0 (5), 127.0 (4), 148.1 (16), 150.0 (16), 189.0 (4), 191.0 (3), 206.0 (1), 208.0 (1), 232.8 (4), 234.8 (7), 236.8 (3).

Bis(chloroacetonitrile)silver(I)-tris(perfluoroethyl)trifluorophosphate $\left[\mathbf{A g}(\mathbf{C l}-\mathbf{A C N})_{2}\right][\mathbf{F A P}]$. The reaction of $30.00 \mathrm{~g}(61.97 \mathrm{mmol})$ $\mathrm{Ag}\left[\mathrm{NO}_{3}\right]$ in $60 \mathrm{~mL}$ chloroacetonitrile with $10.53 \mathrm{~g}(61.97 \mathrm{mmol})$ $\mathrm{K}[\mathrm{FAP}]$ in $15 \mathrm{~mL}$ chloroacetonitrile yielded $30.66 \mathrm{~g}$ (43.56 mmol, $70.30 \%)\left[\mathrm{Ag}(\mathrm{Cl}-\mathrm{ACN})_{2}\right][\mathrm{FAP}]$ as a pale yellowish liquid. ${ }^{1} \mathrm{H}$ NMR (400 MHz, $\left.\mathrm{d}_{4}-\mathrm{MeOD}\right): \delta / \mathrm{ppm}=4.41(\mathrm{~s}, 4 \mathrm{H}) .{ }^{13} \mathrm{C} \mathrm{NMR}(101 \mathrm{MHz}$, $\left.\mathrm{d}_{4}-\mathrm{MeOD}\right): \delta / \mathrm{ppm}=25.16\left(\mathrm{~s}, 2 \mathrm{C}, \mathrm{CH}_{2} \mathrm{Cl}\right), 116.95(\mathrm{~s}, 2 \mathrm{C}, \mathrm{CN}), 121.40$ (m, 6C, $3 \mathrm{C}_{2} \mathrm{~F}_{5}$ ). ${ }^{19} \mathrm{~F}$ NMR (376 MHz, $\left.\mathrm{d}_{4}-\mathrm{MeOD}\right): \delta / \mathrm{ppm}=-117.34$ (md, ${ }^{2} J_{\mathrm{F}, \mathrm{P}}=97.80 \mathrm{~Hz}, 4 \mathrm{~F}, 2 \mathrm{CF}_{2}$ ), $-116.76\left(\mathrm{md},{ }^{2} J_{\mathrm{F}, \mathrm{P}}=83.13 \mathrm{~Hz}, 2 \mathrm{~F}\right.$, $\left.1 \mathrm{CF}_{2}\right),-89.07\left(\mathrm{md},{ }^{1} J_{\mathrm{F}, \mathrm{P}}=901.23 \mathrm{~Hz}, 2 \mathrm{~F}, \mathrm{PF}_{2}\right),-83.12(\mathrm{~m}, 6 \mathrm{~F}$, $\left.2 \mathrm{CF}_{3}\right),-81.42\left(\mathrm{~m}, 3 \mathrm{~F}, 1 \mathrm{CF}_{3}\right),-45.73\left(\mathrm{md},{ }^{1} J_{\mathrm{F}, \mathrm{P}}=889.95 \mathrm{~Hz}, 1 \mathrm{~F}, \mathrm{PF}\right)$. ${ }^{31} \mathrm{P}$ NMR (162 MHz, d $\left.-\mathrm{MeOD}\right): \delta / \mathrm{ppm}=-147.09\left(\mathrm{mtd},{ }^{1} J_{\mathrm{P}, \mathrm{F}}=\right.$ $\left.{ }^{1} J_{\mathrm{P}, \mathrm{F}}=896.34 \mathrm{~Hz}, 1 \mathrm{P}\right) .{ }^{1} \mathrm{H}$ qNMR (400 MHz, d ${ }_{4} \mathrm{MeOD}, 1,3-$ bis(trifluoromethyl)benzene): $n(\mathrm{Cl}-\mathrm{ACN})=2.02$. ESI-MS $(\mathrm{MeOH}$, negative mode): $m / z=118.9$ (81\%), 206.9 (14), 345.0 (43), 443.8 (18), 444.4 (100), 445.4 (90), 446.2 (57). ESI-MS (MeOH, positive mode): $m / z=106.8$ (100\%), 108.9 (97), 124.0 (17), 126.0 (16), 139.0 (15), 141.0 (16), 182.0 (11), 184.0 (14), 186.0 (4), 232.9 (16), 234.9 (27), 236.8 (14). ICP-AES $\left(\mathrm{H}_{2} \mathrm{O}\right): n(\mathrm{Ag}) / n(\mathrm{P})=1.04$.

Bis(acrylonitrile)silver(I)-tris(perfluoroethyl)trifluorophosphate $\left[\mathbf{A g}(\operatorname{acryl}-\mathbf{C N})_{2}\right][\mathbf{F A P}]$. The reaction of $10.53 \mathrm{~g}(61.97 \mathrm{mmol})$ $\mathrm{Ag}\left[\mathrm{NO}_{3}\right]$ in $30 \mathrm{~mL}$ acrylonitrile with $30.00 \mathrm{~g}(61.97 \mathrm{mmol})$ $\mathrm{K}[\mathrm{FAP}]$ in $30 \mathrm{~mL}$ acrylonitrile yielded $20.63 \mathrm{~g}(31.31 \mathrm{mmol}$, $50.52 \%)$ [Ag(acryl-CN $\left.)_{2}\right][\mathrm{FAP}]$ as a colorless solid. mp: $44{ }^{\circ} \mathrm{C}$. ${ }^{1} \mathrm{H}$ NMR (400 MHz, d $\left.{ }_{4}-\mathrm{MeOD}\right): \delta / \mathrm{ppm}=5.84\left(\mathrm{dd},{ }^{3} J_{\mathrm{H}, \mathrm{H}}=\right.$ $\left.17.99 \mathrm{~Hz},{ }^{3} J_{\mathrm{H}, \mathrm{H}}=11.59 \mathrm{~Hz}, 2 \mathrm{H},-\mathrm{CH}=\mathrm{CH}_{2}\right), 6.16\left(\mathrm{~d},{ }^{3} J_{\mathrm{H}, \mathrm{H}}=\right.$ $11.59 \mathrm{~Hz}, 2 \mathrm{H},-\mathrm{CH}=\mathrm{CH}$ cis $), 6.28\left(\mathrm{~d},{ }^{3} J_{\mathrm{H}, \mathrm{H}}=17.99 \mathrm{~Hz}, 2 \mathrm{H}\right.$, $-\mathrm{CH}=\mathrm{CH} H$ trans $).{ }^{13} \mathrm{C}$ NMR (101 MHz, d $\left.\mathrm{d}_{4}-\mathrm{MeOD}\right): \delta / \mathrm{ppm}=$ 107.78 (s, 2C, CH), 118.58 (s, 2C, CN), $121.73\left(\mathrm{~m}, 6 \mathrm{C}, 3 \mathrm{C}_{2} \mathrm{~F}_{5}\right.$ ), 140.05 (s, 2C, $\mathrm{CH}_{2}$ ). ${ }^{19} \mathrm{~F}$ NMR (376 $\left.\mathrm{MHz}, \mathrm{d}_{4}-\mathrm{MeOD}\right): \delta / \mathrm{ppm}=$ $-117.46\left(\mathrm{md},{ }^{2} J_{\mathrm{F}, \mathrm{P}}=97.42 \mathrm{~Hz}, 4 \mathrm{~F}, 2 \mathrm{CF}_{2}\right),-116.87\left(\mathrm{md},{ }^{2} J_{\mathrm{F}, \mathrm{P}}=\right.$ $\left.82.75 \mathrm{~Hz}, 2 \mathrm{~F}, 1 \mathrm{CF}_{2}\right),-89.02\left(\mathrm{md},{ }^{1} J_{\mathrm{F}, \mathrm{P}}=902.36 \mathrm{~Hz}, 2 \mathrm{~F}, \mathrm{PF}_{2}\right.$ ), $-83.17\left(\mathrm{~m}, 6 \mathrm{~F}, 2 \mathrm{CF}_{3}\right),-81.46\left(\mathrm{~m}, 3 \mathrm{~F}, 1 \mathrm{CF}_{3}\right),-45.79\left(\mathrm{md},{ }^{1} J_{\mathrm{F}, \mathrm{P}}=\right.$ $889.95 \mathrm{~Hz}, 1 \mathrm{~F}, \mathrm{PF}) .{ }^{31} \mathrm{P}$ NMR $\left(162 \mathrm{MHz}, \mathrm{d}_{4}-\mathrm{MeOD}\right): \delta / \mathrm{ppm}=$ $-147.30\left(\mathrm{mtd},{ }^{1} J_{\mathrm{P}, \mathrm{F}}={ }^{1} J_{\mathrm{P}, \mathrm{F}}=899.64 \mathrm{~Hz}, 1 \mathrm{P}\right) .{ }^{1} \mathrm{H}$ qNMR $(400 \mathrm{MHz}$, $\mathrm{d}_{4}$-MeOD, 1,3-bis(trifluoromethyl)benzene): $n(\mathrm{ACN})=2.01$. ESI-MS (MeOH, negative mode): $m / z=118.9$ (82\%), 207.1 (13), 345.0 (42), 443.8 (13), 444.5 (100), 445.4 (91), 446.2 (58). ESI-MS ( $\mathrm{MeOH}$, positive mode): $m / z=106.8$ (100\%), 108.7 (93), 123.9 (14), 126.0 (13), 159.9 (40), 162.1 (38), 213.0 (13), 215.0 (12), 232.8 (7), 234.8 (12), 236.9 (6), 285.8 (7), 287.8 (12), 289.8 (6). ICP-AES $\left(\mathrm{H}_{2} \mathrm{O}\right): n(\mathrm{Ag}) / n(\mathrm{P})=1.03$.

Bis(pyridine)silver(I)-tris(perfluoroethyl)trifluorophosphate $\left[\mathbf{A g}(\mathbf{p y})_{2}\right][\mathbf{F A P}]$. The reaction of $10.53 \mathrm{~g}(61.97 \mathrm{mmol}) \mathrm{Ag}\left[\mathrm{NO}_{3}\right]$ in
$60 \mathrm{~mL}$ pyridine with $30.00 \mathrm{~g}(61.97 \mathrm{mmol}) \mathrm{K}$ [FAP] in $20 \mathrm{~mL}$ pyridine yielded $34.94 \mathrm{~g}$ (49.14 mmol, 79.30\%) [Ag(py) $\left.)_{2}\right][\mathrm{FAP}]$ as colorless crystals. mp: $81{ }^{\circ} \mathrm{C} .{ }^{1} \mathrm{H}$ NMR (400 MHz, $\mathrm{d}_{4}$-MeOD): $\delta / \mathrm{ppm}=7.60(\mathrm{~m}, 4 \mathrm{H}, \mathrm{N}-\mathrm{CH}-\mathrm{CH}), 8.02(\mathrm{~m}, 2 \mathrm{H}, \mathrm{N}-\mathrm{CH}-\mathrm{CH}-\mathrm{CH})$, $8.65(\mathrm{~m}, 4 \mathrm{H}, \mathrm{N}-\mathrm{CH}) .{ }^{13} \mathrm{C} \mathrm{NMR}\left(101 \mathrm{MHz}, \mathrm{d}_{4}-\mathrm{MeOD}\right): \delta / \mathrm{ppm}=$ $121.71\left(\mathrm{~m}, 6 \mathrm{C}, 3 \mathrm{C}_{2} \mathrm{~F}_{5}\right), 126.59$ (s, 4C, N-CH-CH), 140.25 (s, 2C, $\mathrm{N}-\mathrm{CH}-\mathrm{CH}-\mathrm{CH}), 152.71$ (s, 4C, N-CH). ${ }^{19} \mathrm{~F}$ NMR $(376 \mathrm{MHz}$, $\mathrm{d}_{4}$-MeOD): $\delta / \mathrm{ppm}=-117.43\left(\mathrm{md},{ }^{2} J_{\mathrm{F}, \mathrm{P}}=97.42 \mathrm{~Hz}, 4 \mathrm{~F}, 2 \mathrm{CF}_{2}\right.$ ), $-116.83\left(\mathrm{md},{ }^{2} J_{\mathrm{F}, \mathrm{P}}=82.75 \mathrm{~Hz}, 2 \mathrm{~F}, 1 \mathrm{CF}_{2}\right),-88.95\left(\mathrm{md},{ }^{1} J_{\mathrm{F}, \mathrm{P}}=\right.$ $\left.902.74 \mathrm{~Hz}, 2 \mathrm{~F}, \mathrm{PF}_{2}\right),-83.14\left(\mathrm{~m}, 6 \mathrm{~F}, 2 \mathrm{CF}_{3}\right),-81.45(\mathrm{~m}, 3 \mathrm{~F}$, $\left.1 \mathrm{CF}_{3}\right),-45.76\left(\mathrm{md},{ }^{1} J_{\mathrm{F}, \mathrm{P}}=890.70 \mathrm{~Hz}, 1 \mathrm{~F}, \mathrm{PF}\right) .{ }^{31} \mathrm{P} \mathrm{NMR}(162 \mathrm{MHz}$, $\mathrm{d}_{4}$-MeOD): $\delta / \mathrm{ppm}=-147.30\left(\mathrm{mtd},{ }^{1} J_{\mathrm{P}, \mathrm{F}}={ }^{1} J_{\mathrm{P}, \mathrm{F}}=899.69 \mathrm{~Hz}, 1 \mathrm{P}\right)$. ${ }^{1} \mathrm{H}$ qNMR (400 MHz, d $\mathrm{d}_{4}$-MeOD, 1,3-bis(trifluoromethyl)benzene): $n(\mathrm{ACN})=2.00$. ESI-MS $(\mathrm{MeOH}$, negative mode $): m / z=118.9(66 \%)$, 207.0 (9), 345.1 (32), 443.8 (9), 444.5 (100), 445.4 (82). ESI-MS (MeOH, positive mode): $m / z=106.8$ (90\%), 108.8 (86), 185.8 (100), 187.8 (95), 265.0 (41), 267.0 (39). ICP-AES $\left(\mathrm{H}_{2} \mathrm{O}\right): n(\mathrm{Ag}) / n(\mathrm{P})=1.00$.

(Ethylenediamine)silver(I)-tris(perfluoroethyl)trifluorophosphate $[\mathbf{A g}(\mathrm{en})][\mathbf{F A P}]$. $\left[\mathrm{Ag}(\mathrm{ACN})_{4}\right][\mathrm{FAP}](2.02 \mathrm{~g}, 2.81 \mathrm{mmol})$ was dissolved in $5 \mathrm{~mL}$ of acetonitrile under argon atmosphere and light exclusion. Ethylenediamine $(0.17 \mathrm{~g}, 2.81 \mathrm{mmol})$ was added and the reaction mixture was stirred for $1 \mathrm{~h}$. Acetonitrile was removed in vacuo to give $[\mathrm{Ag}(\mathrm{en})][\mathrm{FAP}](1.72 \mathrm{~g}, 2.81 \mathrm{mmol})$ as a beige powder in quantitative yield. mp: $141{ }^{\circ} \mathrm{C} .{ }^{1} \mathrm{H}$ NMR (400 MHz, $\mathrm{d}_{4}$-MeOD): $\delta / \mathrm{ppm}=2.98\left(\mathrm{~s}, 4 \mathrm{H}, \mathrm{CH}_{2}\right), 4.88\left(\mathrm{br} \mathrm{s}, 4 \mathrm{H}, \mathrm{NH}_{2}\right) \cdot{ }^{13} \mathrm{C} \mathrm{NMR}$ (101 MHz, d $\mathrm{d}_{4}$-MeOD): $\delta / \mathrm{ppm}=45.74\left(\mathrm{~s}, 2 \mathrm{C}, \mathrm{CH}_{2}\right), 121.83$ $\left(\mathrm{m}, 6 \mathrm{C}, 3 \mathrm{C}_{2} \mathrm{~F}_{5}\right) .{ }^{19} \mathrm{~F}$ NMR (376 MHz, $\left.\mathrm{d}_{4}-\mathrm{MeOD}\right): \delta / \mathrm{ppm}=-117.44$ (md, ${ }^{2} J_{\mathrm{F}, \mathrm{P}}=97.42 \mathrm{~Hz}, 4 \mathrm{~F}, 2 \mathrm{CF}_{2}$ ), -116.85 (md, ${ }^{2} J_{\mathrm{F}, \mathrm{P}}=82.00 \mathrm{~Hz}, 2 \mathrm{~F}$, $1 \mathrm{CF}_{2}$ ), $-89.03\left(\mathrm{md},{ }^{1} J_{\mathrm{F}, \mathrm{P}}=902.74 \mathrm{~Hz}, 2 \mathrm{~F}, \mathrm{PF}_{2}\right),-83.18\left(\mathrm{~m}, 6 \mathrm{~F}, 2 \mathrm{CF}_{3}\right.$ ), $-81.49\left(\mathrm{~m}, 3 \mathrm{~F}, 1 \mathrm{CF}_{3}\right),-45.83\left(\mathrm{md},{ }^{1} J_{\mathrm{F}, \mathrm{P}}=889.19 \mathrm{~Hz}, 1 \mathrm{~F}, \mathrm{PF}\right)$. ${ }^{31} \mathrm{P}$ NMR (162 MHz, d $\left.-\mathrm{MeOD}\right): \delta / \mathrm{ppm}=-147.25(\mathrm{mtd}$, $\left.{ }^{1} J_{\mathrm{P}, \mathrm{F}}={ }^{1} J_{\mathrm{P}, \mathrm{F}}=899.69 \mathrm{~Hz}, 1 \mathrm{P}\right) .{ }^{1} \mathrm{H}$ qNMR (400 MHz, d ${ }_{4}-\mathrm{MeOD}$, 1,3-bis(trifluoromethyl)benzene): $n(\mathrm{ACN})=1.02$. ESI-MS (MeOH, negative mode): $m / z=118.9$ (76\%), 207.0 (12), 345.1 (39), 443.8 (11), 444.5 (100), 445.4 (88), 446.2 (53). ESI-MS (MeOH, positive mode): $m / z=59.1$ (100\%), 106.9 (61), 108.9 (58), 167.0 (92), 169.0 (83), 184.1 (14), 186.1 (15), 227.1 (29), 229.1 (28), 246.0 (9), $248.0(8)$.

\section{Synthetic procedures for the silver propene complexes}

General procedure for $\left[\mathrm{Ag}(\mathrm{ACN})_{2}\left(\mathrm{C}_{3} \mathrm{H}_{6}\right)_{x}\right][\mathrm{FAP}],\left[\mathrm{Ag}(\mathrm{Cl}-\mathrm{ACN})_{2}\right.$ $\left.\left(\mathbf{C}_{3} \mathbf{H}_{6}\right)_{x}\right][\mathbf{F A P}]$ and $\left[\mathrm{Ag}(\operatorname{acryl}-\mathbf{C N})_{2}\left(\mathbf{C}_{3} \mathbf{H}_{6}\right)_{x}\right][\mathbf{F A P}]$. The propene-free $\mathrm{Ag}[\mathrm{FAP}]$ salts were exposed to a pure propene atmosphere under light exclusion. At a constant propene pressure of 1 bar, the reaction mixture was stirred until a homogenous liquid was formed. The propene silver FAP salts were obtained in quantitative yield.

Bis(acetonitrile)(propene)silver(I)-tris(perfluoroethyl)trifluorophosphate $\left[\mathbf{A g}(\mathbf{A C N})_{2}\left(\mathbf{C}_{3} \mathbf{H}_{6}\right)_{x}\right][\mathbf{F A P}] .{ }^{1} \mathrm{H} \mathrm{NMR}(400 \mathrm{MHz}$, no solvent, capillary with $\left.\mathrm{C}_{6} \mathrm{D}_{6}\right): \delta / \mathrm{ppm}=1.70\left(\mathrm{br} \mathrm{d},{ }^{3} \mathrm{~J}_{\mathrm{H}, \mathrm{H}}=\right.$ $\left.5.60 \mathrm{~Hz}, x \cdot 3 \mathrm{H},=\mathrm{CH}-\mathrm{CH}_{3}\right), 1.90\left(\mathrm{~s}, 6 \mathrm{H}, \mathrm{NC}-\mathrm{CH}_{3}\right), 4.90$ (br d, ${ }^{3} J_{\mathrm{H}, \mathrm{H}}=9.59 \mathrm{~Hz}, x \cdot 1 \mathrm{H},-\mathrm{CH}=\mathrm{CH} H$ cis $), 4.95\left(\mathrm{br} \mathrm{d},{ }^{3} J_{\mathrm{H}, \mathrm{H}}=17.19 \mathrm{~Hz}\right.$, $x \cdot 1 \mathrm{H},-\mathrm{CH}=\mathrm{CH} H$ trans), $6.17(\mathrm{~m}, x \cdot 1 \mathrm{H}, \mathrm{CH}) .{ }^{13} \mathrm{C} \mathrm{NMR}(101 \mathrm{MHz}$, no solvent, capillary with $\left.\mathrm{C}_{6} \mathrm{D}_{6}\right): \delta / \mathrm{ppm}=-0.27\left(\mathrm{~s}, 2 \mathrm{C}, \mathrm{NC}-\mathrm{CH}_{3}\right)$, $18.75\left(\mathrm{~s}, x \cdot 1 \mathrm{C},=\mathrm{CH}-\mathrm{CH}_{3}\right), 101.29\left(\mathrm{~s}, x \cdot 1 \mathrm{C}, \mathrm{CH}_{2}\right), 119.20(\mathrm{~s}, 2 \mathrm{C}, \mathrm{CN})$, $120.43\left(\mathrm{~m}, 6 \mathrm{C}, 3 \mathrm{C}_{2} \mathrm{~F}_{5}\right), 132.23(\mathrm{~s}, x \cdot 1 \mathrm{C}, \mathrm{CH}) .{ }^{19} \mathrm{~F}$ NMR $(376 \mathrm{MHz}$, 
no solvent, capillary with $\left.\mathrm{C}_{6} \mathrm{D}_{6}\right): \delta / \mathrm{ppm}=-116.44\left(\mathrm{br} \mathrm{d},{ }^{2} J_{\mathrm{F}, \mathrm{P}}=\right.$ 98.55 Hz, 4F, 2CF 2 ), -115.94 (br d, ${ }^{2} J_{\mathrm{F}, \mathrm{P}}=82.00 \mathrm{~Hz}, 2 \mathrm{~F}, 1 \mathrm{CF}_{2}$ ), $-88.19\left(\mathrm{br} \mathrm{d},{ }^{1} J_{\mathrm{F}, \mathrm{P}}=903.49 \mathrm{~Hz}, 2 \mathrm{~F}, \mathrm{PF}_{2}\right),-82.60\left(\mathrm{~m}, 6 \mathrm{~F}, 2 \mathrm{CF}_{3}\right)$, $-80.89\left(\mathrm{~m}, 3 \mathrm{~F}, 1 \mathrm{CF}_{3}\right),-44.60$ (md, ${ }^{1} J_{\mathrm{F}, \mathrm{P}}=891.83 \mathrm{~Hz}, 1 \mathrm{~F}, \mathrm{PF}$ ). ${ }^{31} \mathrm{P}$ NMR (162 MHz, no solvent, capillary with $\left.\mathrm{C}_{6} \mathrm{D}_{6}\right): \delta / \mathrm{ppm}=$ -147.04 (quintttd, ${ }^{2} J_{\mathrm{P}, \mathrm{F}}={ }^{2} J_{\mathrm{P}, \mathrm{F}}=93.49 \mathrm{~Hz},{ }^{1} J_{\mathrm{P}, \mathrm{F}}={ }^{1} J_{\mathrm{P}, \mathrm{F}}=899.64 \mathrm{~Hz}, 1 \mathrm{P}$ ).

Bis(chloroacetonitrile)(propene)silver(I)-tris(perfluoroethyl)trifluorophosphate $\left[\mathbf{A g}(\mathbf{C l}-\mathbf{A C N})_{2}\left(\mathbf{C}_{3} \mathbf{H}_{6}\right)_{x}\right][\mathbf{F A P}] .{ }^{1} \mathrm{H}$ NMR $(400 \mathrm{MHz}$, no solvent, capillary with $\left.\mathrm{C}_{6} \mathrm{D}_{6}\right): \delta / \mathrm{ppm}=1.72\left(\mathrm{br} \mathrm{s}, x \cdot 3 \mathrm{H}, \mathrm{CH}_{3}\right)$, $4.03\left(\mathrm{~s}, 6 \mathrm{H}, \mathrm{CH}_{2} \mathrm{Cl}\right), 4.96\left(\mathrm{~m}, x \cdot 2 \mathrm{H},=\mathrm{CH}_{2}\right), 6.17(\mathrm{~m}, x \cdot 1 \mathrm{H}, \mathrm{CH})$. ${ }^{13} \mathrm{C}$ NMR (101 MHz, no solvent, capillary with $\left.\mathrm{C}_{6} \mathrm{D}_{6}\right): \delta / \mathrm{ppm}=$ 19.08 (s, x.1C, $\mathrm{CH}_{3}$ ), 23.63 (s, 2C, $\mathrm{CH}_{2} \mathrm{Cl}$ ), 104.19 (s, x.1C, $\mathrm{CH}_{2}$ ), 116.34 (s, 2C, CN), $118.91\left(\mathrm{~m}, 6 \mathrm{C}, 3 \mathrm{C}_{2} \mathrm{~F}_{5}\right), 134.13(\mathrm{~s}, x \cdot 1 \mathrm{C}, \mathrm{CH})$. ${ }^{19} \mathrm{~F}$ NMR (376 MHz, no solvent, capillary with $\mathrm{C}_{6} \mathrm{D}_{6}$ ): $\delta / \mathrm{ppm}=$ $-116.38\left(\mathrm{br} \mathrm{d},{ }^{2} J_{\mathrm{F}, \mathrm{P}}=98.17 \mathrm{~Hz}, 4 \mathrm{~F}, 2 \mathrm{CF}_{2}\right.$ ), $-115.87\left(\mathrm{br} \mathrm{d},{ }^{2} J_{\mathrm{F}, \mathrm{P}}=\right.$ $84.26 \mathrm{~Hz}, 2 \mathrm{~F}, 1 \mathrm{CF}_{2}$ ), -88.80 (br d, ${ }^{1} J_{\mathrm{F}, \mathrm{P}}=900.48 \mathrm{~Hz}, 2 \mathrm{~F}, \mathrm{PF}_{2}$ ), $-82.51\left(\mathrm{~m}, 6 \mathrm{~F}, 2 \mathrm{CF}_{3}\right),-80.76\left(\mathrm{~m}, 3 \mathrm{~F}, 1 \mathrm{CF}_{3}\right),-44.96\left(\mathrm{md},{ }^{1} J_{\mathrm{F}, \mathrm{P}}=\right.$ 888.07 Hz, 1F, PF). ${ }^{31} \mathrm{P}$ NMR (162 MHz, no solvent, capillary with $\left.\mathrm{C}_{6} \mathrm{D}_{6}\right): \delta / \mathrm{ppm}=-146.35\left(\mathrm{mtd},{ }^{1} J_{\mathrm{P}, \mathrm{F}}={ }^{1} J_{\mathrm{P}, \mathrm{F}}=896.29 \mathrm{~Hz}, 1 \mathrm{P}\right)$.

Bis(acrylonitrile)(propene)silver(I)-tris(perfluoroethyl)trifluorophosphate $\left[\mathrm{Ag}(\text { acryl-CN })_{2}\left(\mathbf{C}_{3} \mathbf{H}_{6}\right)_{x}\right][$ FAP $] .{ }^{1} \mathrm{H}$ NMR $(400 \mathrm{MHz}$, no solvent, capillary with $\left.\mathrm{C}_{6} \mathrm{D}_{6}\right): \delta / \mathrm{ppm}=1.91\left(\mathrm{br} \mathrm{d},{ }^{3} J_{\mathrm{H}, \mathrm{H}}=6.40 \mathrm{~Hz}\right.$, $\left.x \cdot 3 \mathrm{H}, \mathrm{CH}_{3}\right), 5.18\left(\mathrm{~m}, x \cdot 2 \mathrm{H}, \mathrm{H}_{3} \mathrm{C}-\mathrm{CH}=\mathrm{CH}_{2}\right), 5.79\left(\mathrm{dd},{ }^{3} J_{\mathrm{H}, \mathrm{H}}=\right.$ $\left.17.99 \mathrm{~Hz},{ }^{3} \mathrm{~J}_{\mathrm{H}, \mathrm{H}}=11.99 \mathrm{~Hz}, 2 \mathrm{H},=\mathrm{CH}-\mathrm{CN}\right), 6.29\left(\mathrm{~d},{ }^{3} \mathrm{~J}_{\mathrm{H}, \mathrm{H}}=\right.$ $11.99 \mathrm{~Hz}, 2 \mathrm{H}, \mathrm{NC}-\mathrm{CH}=\mathrm{CH} H$ cis $), 6.37\left(\mathrm{~m}, x \cdot 1 \mathrm{H},=\mathrm{CH}-\mathrm{CH}_{3}\right)$, $6.42\left(\mathrm{~d},{ }^{3} J_{\mathrm{H}, \mathrm{H}}=17.99 \mathrm{~Hz}, 2 \mathrm{H}, \mathrm{NC}-\mathrm{CH}=\mathrm{CH} H\right.$ trans $) .{ }^{13} \mathrm{C} \mathrm{NMR}$ (101 MHz, no solvent, capillary with $\left.\mathrm{C}_{6} \mathrm{D}_{6}\right): \delta / \mathrm{ppm}=19.14(\mathrm{~s}, x \cdot 1 \mathrm{C}$, $\mathrm{CH}_{3}$ ), $103.95\left(\mathrm{~s}, x \cdot 1 \mathrm{C}, \mathrm{H}_{3} \mathrm{C}-\mathrm{CH}=\mathrm{CH}_{2}\right), 105.41(\mathrm{~s}, 2 \mathrm{C},=\mathrm{CH}-\mathrm{CN})$, $118.27(\mathrm{~s}, 2 \mathrm{C}, \mathrm{CN}), 120.69\left(\mathrm{~m}, 6 \mathrm{C}, 3 \mathrm{C}_{2} \mathrm{~F}_{5}\right), 133.45(\mathrm{~s}, x \cdot 1 \mathrm{C}$, $\left.=\mathrm{CH}-\mathrm{CH}_{3}\right), 141.47\left(\mathrm{~s}, 2 \mathrm{C}, \mathrm{NC}-\mathrm{CH}=\mathrm{CH}_{2}\right) .{ }^{19} \mathrm{~F}$ NMR $(376 \mathrm{MHz}$, no solvent, capillary with $\left.\mathrm{C}_{6} \mathrm{D}_{6}\right): \delta / \mathrm{ppm}=-116.21\left(\mathrm{br} \mathrm{d},{ }^{2} J_{\mathrm{F}, \mathrm{P}}=\right.$ 98.55 Hz, 4F, 2CF $),-115.70\left(\mathrm{br} \mathrm{d},{ }^{2} J_{\mathrm{F}, \mathrm{P}}=82.37 \mathrm{~Hz}, 2 \mathrm{~F}, 1 \mathrm{CF}_{2}\right.$ ), $-87.96\left(\mathrm{br} \mathrm{d},{ }^{1} J_{\mathrm{F}, \mathrm{P}}=899.35 \mathrm{~Hz}, 2 \mathrm{~F}, \mathrm{PF}_{2}\right),-82.20\left(\mathrm{~m}, 6 \mathrm{~F}, 2 \mathrm{CF}_{3}\right)$, $-80.52\left(\mathrm{~m}, 3 \mathrm{~F}, 1 \mathrm{CF}_{3}\right),-44.47$ (md, $\left.{ }^{1} J_{\mathrm{F}, \mathrm{P}}=891.83 \mathrm{~Hz}, 1 \mathrm{~F}, \mathrm{PF}\right)$. ${ }^{31} \mathrm{P}$ NMR (162 MHz, no solvent, capillary with $\left.\mathrm{C}_{6} \mathrm{D}_{6}\right): \delta / \mathrm{ppm}=$ $-146.84\left(\mathrm{mtd},{ }^{1} J_{\mathrm{P}, \mathrm{F}}={ }^{1} J_{\mathrm{P}, \mathrm{F}}=902.88 \mathrm{~Hz}, 1 \mathrm{P}\right)$.

(Propene)silver(I)-tris(perfluoroethyl)trifluorophosphate $\left[\mathbf{A g}\left(\mathbf{C}_{3} \mathbf{H}_{6}\right)_{x}\right][\mathbf{F A P}]$. Under air and light exclusion, a solution of $\mathrm{Ag}\left[\mathrm{NO}_{3}\right](5.00 \mathrm{~g}, 29.43 \mathrm{mmol})$ in $7 \mathrm{~mL} \mathrm{H}_{2} \mathrm{O}$ was saturated with propene at $0{ }^{\circ} \mathrm{C}$. From this point onwards, propene saturation was maintained throughout the experiment. After addition of $25 \mathrm{~mL} \mathrm{CH}_{2} \mathrm{Cl}_{2}$ and a solution of $\mathrm{K}[\mathrm{FAP}](14.25 \mathrm{~g}, 29.43 \mathrm{mmol})$ in $25 \mathrm{~mL} \mathrm{H}_{2} \mathrm{O}$, the reaction mixture was stirred for $2 \mathrm{~h}$. The organic phase was separated, washed with $\mathrm{H}_{2} \mathrm{O}$ and dried with $\mathrm{Mg}\left[\mathrm{SO}_{4}\right]$. $\mathrm{CH}_{2} \mathrm{Cl}_{2}$ was mainly removed by purging with propene. Alternating drying in high vacuum and purging with propene finally yielded $\left[\mathrm{Ag}\left(\mathrm{C}_{3} \mathrm{H}_{6}\right)_{x}\right][\mathrm{FAP}]$ as a white solid at a propene pressure of 1 bar. Yield: n. d. mp: $53{ }^{\circ} \mathrm{C} .{ }^{1} \mathrm{H}$ NMR (400 MHz, $\left.\mathrm{d}_{4}-\mathrm{MeOD}\right): \delta / \mathrm{ppm}=1.80$ $\left(\mathrm{ddd},{ }^{3} J_{\mathrm{H}, \mathrm{H}}=6.80 \mathrm{~Hz},{ }^{4} J_{\mathrm{H}, \mathrm{H}}={ }^{4} J_{\mathrm{H}, \mathrm{H}}=1.40 \mathrm{~Hz}, x \cdot 3 \mathrm{H}, \mathrm{CH}_{3}\right), 5.00$ (qdd, ${ }^{3} J_{\mathrm{H}, \mathrm{H}}=9.99 \mathrm{~Hz},{ }^{2} J_{\mathrm{H}, \mathrm{H}}={ }^{4} J_{\mathrm{H}, \mathrm{H}}=1.20 \mathrm{~Hz}, x \cdot 1 \mathrm{H},-\mathrm{CH}=\mathrm{CH} H$ cis), $5.07\left(\mathrm{qdd},{ }^{3} J_{\mathrm{H}, \mathrm{H}}=16.79 \mathrm{~Hz},{ }^{4} J_{\mathrm{H}, \mathrm{H}}={ }^{4} J_{\mathrm{H}, \mathrm{H}}=1.35 \mathrm{~Hz}, x \cdot 1 \mathrm{H}\right.$, $-\mathrm{CH}=\mathrm{CH} H$ trans), 6.08 (qdd, ${ }^{3} J_{\mathrm{H}, \mathrm{H}}=16.79 \mathrm{~Hz},{ }^{3} J_{\mathrm{H}, \mathrm{H}}=9.99 \mathrm{~Hz}$, $\left.{ }^{3} J_{\mathrm{H}, \mathrm{H}}=6.40 \mathrm{~Hz}, x \cdot 1 \mathrm{H}, \mathrm{CH}\right) \cdot{ }^{13} \mathrm{C} \mathrm{NMR}\left(101 \mathrm{MHz}, \mathrm{d}_{4}-\mathrm{MeOD}\right): \delta / \mathrm{ppm}=$ $19.74\left(\mathrm{~s}, x \cdot 1 \mathrm{C}, \mathrm{CH}_{3}\right), 109.23\left(\mathrm{~s}, x \cdot 1 \mathrm{C}, \mathrm{CH}_{2}\right), 121.79(\mathrm{~m}, 6 \mathrm{C}$, $3 \mathrm{C}_{2} \mathrm{~F}_{5}$ ), 132.69 (s, $\left.x \cdot 1 \mathrm{C}, \mathrm{CH}\right) .{ }^{19} \mathrm{~F}$ NMR (376 MHz, d $\left.{ }_{4}-\mathrm{MeOD}\right)$ : $\delta / \mathrm{ppm}=-117.51\left(\mathrm{md},{ }^{2} J_{\mathrm{F}, \mathrm{P}}=97.04 \mathrm{~Hz}, 4 \mathrm{~F}, 2 \mathrm{CF}_{2}\right),-116.89$ (md, ${ }^{2} J_{\mathrm{F}, \mathrm{P}}=82.75 \mathrm{~Hz}, 2 \mathrm{~F}, 1 \mathrm{CF}_{2}$ ), $-89.05\left(\mathrm{md},{ }^{1} J_{\mathrm{F}, \mathrm{P}}=902.36 \mathrm{~Hz}\right.$, $\left.2 \mathrm{~F}, \mathrm{PF}_{2}\right),-83.21\left(\mathrm{~m}, 6 \mathrm{~F}, 2 \mathrm{CF}_{3}\right),-81.52\left(\mathrm{~m}, 3 \mathrm{~F}, 1 \mathrm{CF}_{3}\right),-45.86$ (md, $\left.{ }^{1} J_{\mathrm{F}, \mathrm{P}}=888.82 \mathrm{~Hz}, 1 \mathrm{~F}, \mathrm{PF}\right) .{ }^{31} \mathrm{P}$ NMR (162 MHz, d $\left.\mathrm{d}_{4}-\mathrm{MeOD}\right)$ : $\delta / \mathrm{ppm}=-147.22\left(\mathrm{mtd},{ }^{1} J_{\mathrm{P}, \mathrm{F}}={ }^{1} J_{\mathrm{P}, \mathrm{F}}=899.64 \mathrm{~Hz}, 1 \mathrm{P}\right)$. ESI-MS ( $\mathrm{H}_{2} \mathrm{O}$, negative mode): $m / z=60.0$ (45\%), 119.0 (34), 199.3 (31), 255.6 (49), 256.0 (31), 345.3 (17), 444.9 (100), 445.7 (18), 446.1 (19). ESI-MS ( $\mathrm{H}_{2} \mathrm{O}$, positive mode): $\mathrm{m} / \mathrm{z}=106.8$ (100\%), 108.9 (95), 124.9 (6), 127.0 (6), 232.8 (11), 234.8 (19), 236.8 (10). $\operatorname{ICP}-\operatorname{AES}\left(\mathrm{H}_{2} \mathrm{O}\right): n(\mathrm{Ag}) / n(\mathrm{P})=1.06$.

\section{Analysis}

All samples for analysis were either prepared in a glovebox under inert conditions (argon 4.6) or by using Schlenk flasks with an argon or propene atmosphere.

NMR. NMR spectra were recorded on a $400 \mathrm{MHz}$ Jeol ECX 400 spectrometer and referenced for the indicated deuterated solvent. For the liquid propene complexes a sealed capillary with $\mathrm{C}_{6} \mathrm{D}_{6}$ was used. The ligand number was determined by quantitative NMR (qNMR) measurements with 1,3-bis(trifluoromethyl)benzene as internal standard according to the following equation:

$$
\text { Ligand number } x=M_{\mathrm{Ag}[\mathrm{FAP}]} \times\left(\frac{\frac{N_{\mathrm{H}, \mathrm{st}} \times I_{1}}{N_{\mathrm{H}, 1} \times I_{\mathrm{st}}} \times \frac{m_{\mathrm{st}}}{M_{\mathrm{st}}}}{m_{[\mathrm{Ag}(1)][\mathrm{FAP}]}}-\frac{1}{M_{\mathrm{l}}}\right) \text {. }
$$

$N_{\mathrm{H}}$ : number of hydrogen atoms, I: peak integral/area, st: standard and l: ligand.

ESI-MS. ESI-MS spectra were measured using a Q-trap spectrometer from Applied Biosystems/MDS Sciex with an ion spray voltage of $4500 \mathrm{~V}$ and a declustering potential of 70-80 V.

ICP-AES. ICP-AES measurements were carried out on a Spectra CIROS-CCD spectrometer that was calibrated with standard solutions of $\mathrm{Ag}, \mathrm{P}$ and $\mathrm{K}$ prior to each measurement. Silver salts insoluble in water were dissolved by addition of a small amount of $\mathrm{H}\left[\mathrm{NO}_{3}\right]$.

$\mathrm{X}$-ray crystallography. Crystals were obtained from a solution of the respective silver complex in a small excess of ligand by slow evaporation. Crystallographic data were recorded on a Bruker Kappa APEX $2 \mathrm{I} \mu \mathrm{S}$ Duo ([Ag(ACN/py $\left.\left.)_{2}\right][\mathrm{FAP}]\right)$ and a Bruker Smart APEX 2 ([Ag(ACN)4][FAP]) diffractometer with $\operatorname{MoK}_{\alpha}$ radiation $(\lambda=0.71073 \AA)$ at $100 \mathrm{~K}$. Semiempirical absorption corrections from multiple measurements of equivalent reflections were applied (SADABS $2014 / 4^{37}$ for [Ag(ACN/py)2][FAP] and SADABS $2012 / 1^{38}$ for $\left.\left[\mathrm{Ag}(\mathrm{ACN})_{4}\right][\mathrm{FAP}]\right)$. Structures were solved by direct methods (SHELXTL NT 6.12). ${ }^{39}$ Refinements were conducted with full-matrix least squares methods on $F^{2}$ for all non-hydrogen atoms anisotropically (SHELXL-2014/6). ${ }^{40}$ The $[\mathrm{FAP}]^{-}$moiety was disordered with two different orientations around the central $\mathrm{P}$ atom for $\left[\mathrm{Ag}(\mathrm{ACN})_{2}\right][\mathrm{FAP}]$ and $\left[\mathrm{Ag}(\mathrm{ACN})_{4}\right][\mathrm{FAP}]$. The refined site occupancies for the two orientations were $53.6(1) \%$ and $46.4(1) \%$ for $\left[\mathrm{Ag}(\mathrm{ACN})_{2}\right][\mathrm{FAP}]$ and $53.5(2) \%$ and $46.5(2) \%$ for $\left[\mathrm{Ag}(\mathrm{ACN})_{4}\right][\mathrm{FAP}]$. Similarity and pseudo-isotropic restraints (SIMU, ISOR) were applied to the anisotropic displacement ellipsoids of some of the disordered atoms. The distances C13-C14 and C13A-C14A for $\left[\mathrm{Ag}(\mathrm{ACN})_{4}\right][\mathrm{FAP}]$ were restrained to be of approximately the same length using an appropriate 
similarity restraint (SADI). All hydrogen atoms were placed in positions of optimized geometry. The isotropic displacement parameters of all hydrogen atoms were tied to those of their corresponding carrier atoms by a factor of 1.2 or 1.5. Graphics were created with SHELXTL NT 6.12. ${ }^{39}$

Propene pressure for liquefaction. A small amount of the solid silver salts was placed into a NMR pressure tube from Wilmad LabGlass (522-PV-7), whose temperature was controlled with a heated water bath (Fig. 1). After evacuation the propene pressure was very slowly increased in small steps until liquefaction of the solid salt could visually be observed. The current pressure was monitored with the pressure sensor GMSD 35 BAE (GHM Messtechnik) with a resolution of 10 mbar.

Melting point. Melting points were determined on a SMP10 from Bibby Scientific Limited and by differential scanning calorimetry (DSC) on a Netzsch DSC 204F1 instrument. For the DSC measurements samples were weighed into an aluminum or pressure resistant $\mathrm{Cr} / \mathrm{Ni}$ steel $\left(\left[\mathrm{Ag}\left(\mathrm{C}_{3} \mathrm{H}_{6}\right)_{x}\right][\mathrm{FAP}]\right)$ crucible and heated and cooled three times with $5 \mathrm{~K} \mathrm{~min}^{-1}$.

Thermal stability. Onset decomposition temperatures were obtained from thermogravimetric analyses (TGA) using a Setsys Evolution (Setaram Instrumentation). The silver complexes were placed into an aluminum oxide crucible and heated with $5 \mathrm{~K} \mathrm{~min}^{-1}$ while being purged with helium (flow rate $50 \mathrm{~mL} \mathrm{~min}^{-1}$ ).
Density. Density measurements were performed using a DSA $5000 \mathrm{M}$ instrument from Anton Paar (liquid samples) and a Pycnomatic ATC helium pycnometer from Porotec (solid samples).

Gas solubility. Propene and propane solubilities of the silver salts were measured with an isochoric gas solubility cell (iGSC, single gas and pseudo single gas measurements) and by qNMR spectroscopy (mixed gas measurements).

The key part of the iGSC apparatus (Fig. 1) is the reactor R-2 and the feed tank R-1, two stainless steel autoclaves, equipped with two magnetic stirrers. Temperature is set with a thermostatically controlled cabinet TC $135 \mathrm{~S}$ from Aqualytic and recorded by two Pt-100 thermocouples TI-1 and TI-2. The pressure is recorded with a digital pressure sensor MSD-6BAE (PI-1) from GHM Messtechnik with a resolution of 1 mbar. Both autoclaves can be separated or connected via valve V-6. Vacuum, argon for purging, exhaust and pressure relief as well as propene and propane is provided via valve V-5. After placing a known amount of a sample into R-1, the stirrers were switched on with a stirrer speed of $600 \mathrm{rpm}$ and the apparatus was evacuated. V-6 was closed and R-1 was pressurized with propene. After equilibrating for at least $1 \mathrm{~h}, \mathrm{~V}-6$ was opened and a continuously decreasing pressure due to gas absorption was observed. The measurement was stopped when the pressure decrease was $<2 \mathrm{mbar}^{-1}$. Equilibration time varied between several hours and some days
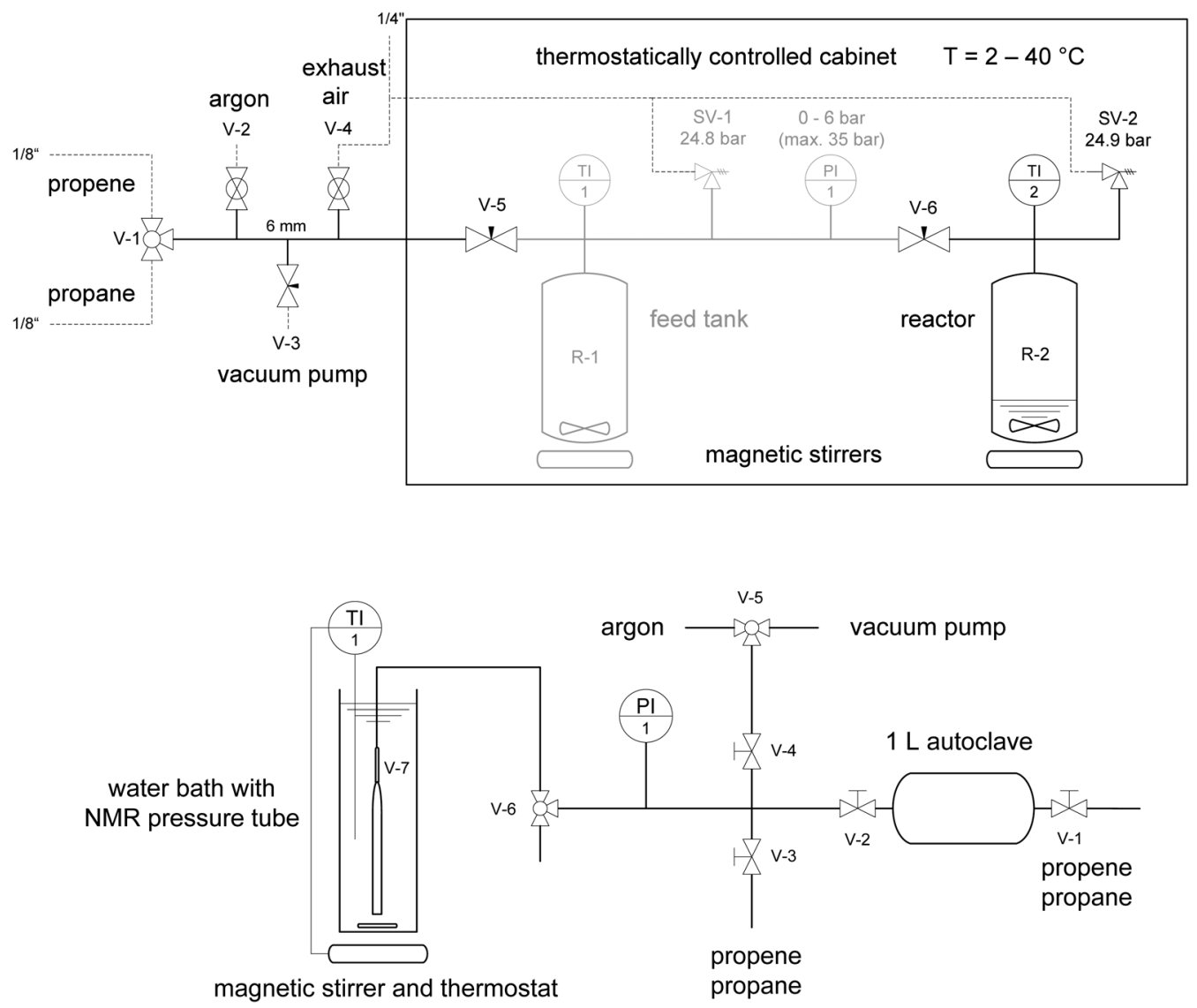

Fig. 1 Isochoric gas solubility cell (top) and pressure NMR sample preparation setup (bottom). 
depending on the investigated silver salt. The procedure was repeated with subsequently increased pressures until a final pressure of 5.5-6.0 bar was reached. Before starting the propane measurements, a low propene pressure of $0.11-0.12$ bar was adjusted in order to form the liquid propene complex for propane absorption first. The propane solubility measurement (pseudo single gas solubility) was then carried out as described previously for propene. Gas solubilities were calculated from the initial (before absorption) and final (equilibrium absorption) amount of gas in the gas phase, via the virial equation with two virial coefficients. Volume dilatation by liquefaction and gas uptake of the sample as well as volume change of the gas phase by opening and closing V-6 were taken into account.

Samples for gas solubility determination by qNMR spectroscopy were prepared with the setup shown in Fig. 1. A pressure/ vacuum NMR tube with integrated valve (V-7) from Wilmad LabGlass (522-PV-7) was used. Temperature control was achieved with a thermostat regulated water bath. The pressure was observed with a GMSD 35 BAE pressure sensor from GHM Messtechnik with a resolution of $10 \mathrm{mbar}$. The huge $1 \mathrm{~L}$ autoclave compared to the small NMR tube ensured a constant pressure and composition of the gas phase despite absorption. Gas and vacuum supply was enabled through V-1-V-5. Sample preparation started with filling the NMR tube with the silver salt and evacuation of the whole rig. After adjusting the intended propene pressure in the NMR tube, it was pressurized three times with the intended propane pressure by quickly opening and closing V-7. During the whole absorption process, the sample was vigorously mixed through shaking. The gas mixture, previously adjusted in the NMR tube, was then prepared in the $1 \mathrm{~L}$ autoclave and contacted with the NMR tube. Equilibrium was allowed to reach overnight. For the qNMR measurements the NMR sample chamber was preheated to the target temperature first. Shimming was achieved by gradient shimming with a similar sample containing $\mathrm{d}_{4}-\mathrm{MeOD}$ and by shimming on FID manually with the actual sample afterwards. Gas solubilities were calculated from the ratio of the propene and propane peak areas to the ligand peak area, which acted as internal standard.

Diffusion coefficients. Diffusion coefficients of propene and propane in the liquid silver complexes were obtained by ${ }^{1} \mathrm{H}$ NMR PFG (pulsed field gradient) measurements ${ }^{41-46}$ with a BPP-STE-LED (bipolar pulse pairs stimulated echo longitudinal eddy current delay) pulse sequence. ${ }^{47,48}$ The same samples as for the qNMR determination of the gas solubilities were used. The Stejskal-Tanner equation ${ }^{47,49}$ generalized for a bipolar sinusoidal magnetic field gradient links the diffusion coefficient $D$ to the parameters of the experiment:

$$
\ln \frac{S}{S_{0}}=-D g^{2} \gamma^{2} \delta^{2} \times \frac{4}{\pi^{2}}\left(\Delta-\frac{5 \delta}{16}-\frac{\tau}{2}\right)
$$

$\tau$ is the longitudinal eddy current delay time $(2.0256 \mathrm{~ms}), \delta$ is the duration of the magnetic field gradient (1,2 ms), $\Delta$ represents the diffusion time (100 $\mathrm{ms})$ and $\gamma$ stands for the gyromagnetic ratio. $S$ and $S_{0}$ are the spin echo signal intensities of the respective peak with and without magnetic field gradient. $g$ is the magnitude of the magnetic field gradient and was varied from $10 \mathrm{mT} \mathrm{m}^{-1}$ to
$610 \mathrm{mT} \mathrm{m}^{-1}$ in steps of $30 \mathrm{mT} \mathrm{m}^{-1} . D$ was then calculated from the slope of the plot of $\ln S / S_{0} v s . g^{2}$. The magnetic field gradient was calibrated using reference measurements with $\mathrm{H}_{2} \mathrm{O}$. Reference values for the diffusion coefficient of $\mathrm{H}_{2} \mathrm{O}$ were taken from Holz et al. ${ }^{50}$

\section{Results and discussion}

\section{Synthesis and characterization}

There are few examples in the literature describing the synthesis of Ag[FAP] salts. ${ }^{36,51}$ All have in common that they use $\mathrm{H}[\mathrm{FAP}]$ or tris(perfluoroethyl)difluorophosphorane $\left(\left[\mathrm{PF}_{2}\left(\mathrm{C}_{2} \mathrm{~F}_{5}\right)_{3}\right]\right)$ as the source for the $[\mathrm{FAP}]^{-}$ion: either the neutralization reaction between $\mathrm{H}[\mathrm{FAP}]$ and a basic silver salt, such as $\mathrm{Ag}_{2}\left[\mathrm{CO}_{3}\right]$ or $\mathrm{Ag}_{2} \mathrm{O}$, or the fluorine affinity of $\left[\mathrm{PF}_{2}\left(\mathrm{C}_{2} \mathrm{~F}_{5}\right)_{3}\right]$ in contact with $\mathrm{AgF}$ or $\mathrm{Ag}\left[\mathrm{BF}_{4}\right]$ is used. An unsuccessful attempt to yield $\mathrm{Ag}[\mathrm{FAP}]$ from $\mathrm{Ag}\left[\mathrm{PF}_{6}\right]$ and $\mathrm{K}[\mathrm{FAP}]$ by precipitating $\mathrm{K}\left[\mathrm{PF}_{6}\right]$ in $\mathrm{ACN}$ has also been reported. ${ }^{36}$ The high vapor pressures of $\left[\mathrm{PF}_{2}\left(\mathrm{C}_{2} \mathrm{~F}_{5}\right)_{3}\right]$ and $\mathrm{H}[\mathrm{FAP}]$ as well as the possibility of residual HF prompted us to develop synthesis routes starting from K[FAP].

We obtained a series of $\mathrm{Ag}[\mathrm{FAP}]$ complexes (Fig. 2) with $\mathrm{N}$-coordinating ligands with different coordination strength by replacing $\mathrm{Ag}\left[\mathrm{PF}_{6}\right]$ with $\mathrm{Ag}\left[\mathrm{NO}_{3}\right]$ and precipitation of $\mathrm{K}\left[\mathrm{NO}_{3}\right]$ (Scheme 1). Subsequent drying in vacuum yielded the respective silver complexes. Due to the high water solubility of $\mathrm{Ag}\left[\mathrm{NO}_{3}\right]$ and $\mathrm{K}\left[\mathrm{NO}_{3}\right]$ and the hydrophobicity of the $[\mathrm{FAP}]^{-}$anion, substrate residues could be removed by washing with water. In addition, synthesis costs could be reduced with $\mathrm{Ag}\left[\mathrm{NO}_{3}\right]$ being the cheapest silver salt available.

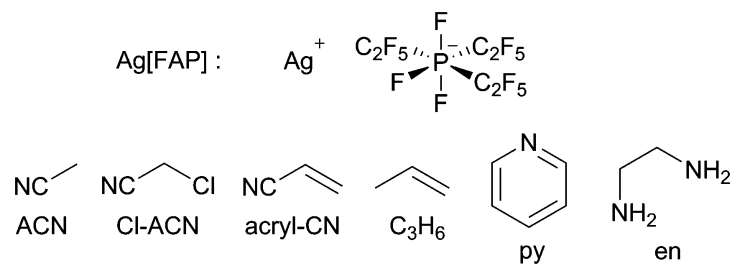

Fig. 2 Structural formulae and abbreviations of silver tris(perfluoroethyl)trifluorophosphate and the employed ligands.

$$
\begin{aligned}
& \mathrm{Ag}\left[\mathrm{NO}_{3}\right]+\mathrm{K}[\mathrm{FAP}] \stackrel{\text { ligand } \mathrm{L}}{\longrightarrow}\left[\mathrm{Ag}(\mathrm{L})_{\mathrm{x}}\right][\mathrm{FAP}]+\mathrm{K}\left[\mathrm{NO}_{3}\right] \downarrow \\
& \mathrm{L}=\mathrm{ACN}, \mathrm{Cl}-\mathrm{ACN} \text {, acryl-CN, py } \\
& {\left[\mathrm{Ag}(\mathrm{ACN})_{4}\right][\mathrm{FAP}]+\text { en } \longrightarrow[\mathrm{Ag}(\mathrm{en})][\mathrm{FAP}]+4 \mathrm{ACN}}
\end{aligned}
$$

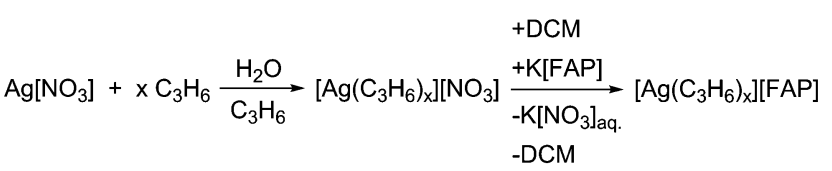

$$
\begin{aligned}
& {\left[\mathrm{Ag}(\mathrm{L})_{\mathrm{x}}\right][\mathrm{FAP}]+\mathrm{y} \mathrm{C}_{3} \mathrm{H}_{6} \longrightarrow\left[\mathrm{Ag}(\mathrm{L})_{\mathrm{x}}\left(\mathrm{C}_{3} \mathrm{H}_{6}\right)_{y}\right][\mathrm{FAP}]} \\
& \mathrm{L}=\mathrm{ACN}, \mathrm{Cl}-\mathrm{ACN} \text {, acryl-CN, py, en }
\end{aligned}
$$

Scheme 1 Synthetic pathways to the silver FAP salt complexes. 
Other solvents like methanol, isopropanol, diethyl ether, 1,4dioxane, acetone, tetrahydrofuran, dichloromethane, chloroform, ethyl acetate, dimethyl sulfoxide, dimethylformamide, ethylene glycol, polyethylene glycol 200, toluene, 1-hexene, acetic acid, cyclooctadiene and ethylenediamine were not found to be suitable for this reaction. These solvents do not provide enough solubility for the reactants along with insolubility of $\mathrm{K}\left[\mathrm{NO}_{3}\right]$ and compatibility for the product. However, $[\mathrm{Ag}(\mathrm{en})][\mathrm{FAP}]$ could be obtained by substitution of acetonitrile by the strongly coordinating en ligand in quantitative yield. The respective liquid propene complexes were formed by applying different propene pressures as will be discussed in detail below.

Neat $\left[\mathrm{Ag}\left(\mathrm{C}_{3} \mathrm{H}_{6}\right)_{x}\right][\mathrm{FAP}]$ was synthesized following a modified approach of Huang et al.: ${ }^{23,52}$ an aqueous solution of silver nitrate was saturated with propene. By addition of DCM and $\mathrm{K}[\mathrm{FAP}]$ a biphasic reaction system formed. The $\left[\mathrm{Ag}\left(\mathrm{C}_{3} \mathrm{H}_{6}\right)_{x}\right][\mathrm{FAP}]$ complex could then be extracted into the DCM phase, from which it was isolated afterwards, while $\mathrm{K}\left[\mathrm{NO}_{3}\right]$ is found in the aqueous phase. Attempts to isolate neat Ag[FAP] led in all cases to the decomposition of the respective silver salts.

The successful formation of all prepared silver salt complexes was verified by ${ }^{1} \mathrm{H},{ }^{13} \mathrm{C},{ }^{31} \mathrm{P},{ }^{19} \mathrm{~F}$ NMR, ${ }^{1} \mathrm{H}$ qNMR, ESI-MS and ICP-AES measurements. The organic ligands including propene and the number of ligands per mole of silver were identified by ${ }^{1} \mathrm{H},{ }^{13} \mathrm{C}$ and ${ }^{1} \mathrm{H}$ qNMR experiments with 1,3-bis(trifluoromethyl)benzene as internal standard. The FAP anion could well be detected by its characteristic splitting pattern in the ${ }^{19} \mathrm{~F}$ and ${ }^{31} \mathrm{P}$ NMR spectra. Solely the meridional conformation was observed. The presence of silver and the absence of potassium and nitrate were verified by ICP-AES analysis either by direct detection or from the ratio of the amount of silver and phosphorous atoms. ESI-MS spectra were used to confirm the previous results.

In addition, the molecular and crystal structures of $\left[\mathrm{Ag}(\mathrm{ACN})_{4}\right][\mathrm{FAP}],\left[\mathrm{Ag}(\mathrm{ACN})_{2}\right][\mathrm{FAP}]$ and $\left[\mathrm{Ag}(\mathrm{py})_{2}\right][\mathrm{FAP}]$ as representative examples for $\mathrm{Ag}[\mathrm{FAP}]$ complexes with different coordination spheres and properties were determined by single crystal X-ray analyses (Fig. 3). Selected bond distances and angles are displayed in Table $1 .\left[\mathrm{Ag}(\mathrm{ACN})_{2}\right][\mathrm{FAP}]$ and $\left[\mathrm{Ag}(\mathrm{py})_{2}\right][\mathrm{FAP}]$ with two ligands form monoclinic crystals with the space groups $P 2_{1} / n$ and $P 2_{1} / c$, respectively. The ligands in both complexes are arranged in an almost linear way with $\mathrm{N} 1-\mathrm{Ag}-\mathrm{N} 2$ angles of $176.75(6)^{\circ}$ and $174.80(5)^{\circ}$ and $\mathrm{Ag}-\mathrm{N}$ distances around $2.1 \AA$ which is the typical range of silver acetonitrile complexes with weakly coordinating anions. ${ }^{53-55}$ Weak interactions of silver with the anion are mainly detected via an axial fluorine atom for both $\left[\mathrm{Ag}(\mathrm{ACN})_{2}\right][\mathrm{FAP}]$

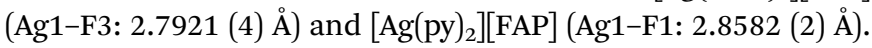
In contrast to $\left[\mathrm{Ag}(\mathrm{ACN})_{2}\right][\mathrm{FAP}],\left[\mathrm{Ag}(\mathrm{py})_{2}\right][\mathrm{FAP}]$ forms a dimeric arrangement of the cationic $\left[\mathrm{Ag}(\mathrm{py})_{2}\right]$ moiety in the crystal lattice with a light offset arrangement of the pyridine rings and a twisting angle of the aromatic ring planes of $10.76^{\circ}$. The distance between the centroids of two adjacent pyridine rings was found to be $3.670 \AA$ and thus lies in the range for $\pi-\pi$ interactions. ${ }^{56}$ The $\mathrm{Ag}-\mathrm{Ag}$ distance of 3.4754 (3) $\AA$ is smaller than twice the van der Waals radius of silver determined by Alvarez, ${ }^{57}$ but slightly longer than many reported $\mathrm{Ag}-\mathrm{Ag}$ bond lengths for dimeric structures, which are often between 2.6 and $3.4 \AA^{58}$
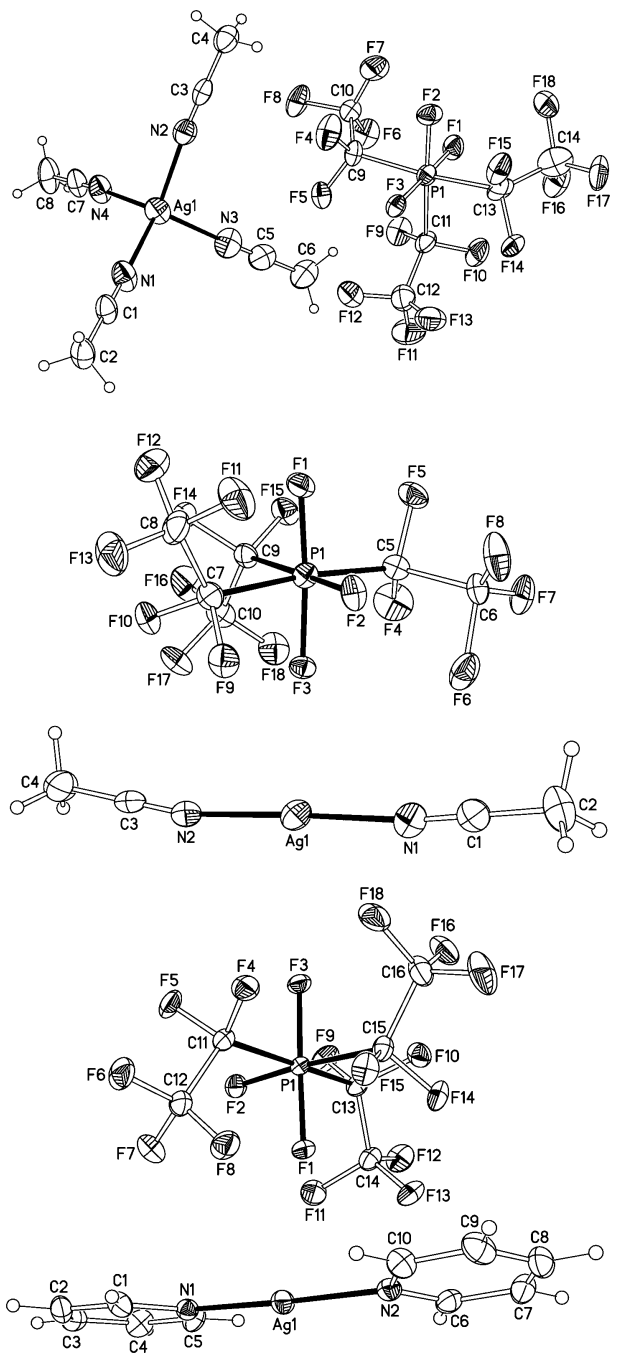

Fig. 3 Crystal structures of $\left[\mathrm{Ag}(\mathrm{ACN})_{4}\right][\mathrm{FAP}],\left[\mathrm{Ag}(\mathrm{ACN})_{2}\right][\mathrm{FAP}]$ and $\left[\mathrm{Ag}(\mathrm{py})_{2}\right][\mathrm{FAP}]$. Thermal ellipsoids are drawn at a level of $50 \%$ probability.

Table 1 Selected bond lengths and angles of $\left[\mathrm{Ag}(\mathrm{ACN})_{4}\right][\mathrm{FAP}],\left[\mathrm{Ag}(\mathrm{ACN})_{2}\right][\mathrm{FAP}]$ and $\left[\mathrm{Ag}(\mathrm{py})_{2}\right][\mathrm{FAP}]$

\begin{tabular}{llll}
\hline & {$\left[\mathrm{Ag}(\mathrm{ACN})_{4}\right][\mathrm{FAP}]$} & {$\left[\mathrm{Ag}(\mathrm{ACN})_{2}\right][\mathrm{FAP}]$} & {$\left[\mathrm{Ag}(\mathrm{py})_{2}\right][\mathrm{FAP}]$} \\
\hline $\mathrm{Ag} 1-\mathrm{N} 1 / \AA$ & $2.269(3)$ & $2.093(2)$ & $2.104(2)$ \\
$\mathrm{Ag} 1-\mathrm{N} 2 / \AA$ & $2.283(3)$ & $2.098(2)$ & $2.108(2)$ \\
$\mathrm{Ag} 1-\mathrm{N} 3 / \AA$ & $2.290(3)$ & - & - \\
$\mathrm{Ag} 1-\mathrm{N} 4 / \AA$ & $2.296(3)$ & - & - \\
$\mathrm{N} 1-\mathrm{Ag} 1-\mathrm{N} 2{ }^{\circ}$ & $113.0(1)$ & $176.75(6)$ & - \\
$\mathrm{N} 1-\mathrm{Ag} 1-\mathrm{N} 3{ }^{\circ}$ & $109.6(2)$ & - & - \\
$\mathrm{N} 1-\mathrm{Ag} 1-\mathrm{N} 4{ }^{\circ}$ & $113.7(1)$ & - & - \\
$\mathrm{N} 2-\mathrm{Ag} 1-\mathrm{N} 3 /^{\circ}$ & $107.5(1)$ & - & - \\
$\mathrm{N} 2-\mathrm{Ag} 1-\mathrm{N} 4{ }^{\circ}$ & $108.2(1)$ & - & - \\
$\mathrm{N} 3-\mathrm{Ag} 1-\mathrm{N} 4 /^{\circ}$ & $104.2(2)$ & - & \\
\end{tabular}

The Ag[FAP] complex with four ligands, $\left[\mathrm{Ag}(\mathrm{ACN})_{4}\right][\mathrm{FAP}]$, crystallizes in the orthorhombic space group Pbca. The acetonitrile ligands form a slightly distorted tetrahedral coordination sphere with a mean $\mathrm{N}-\mathrm{Ag}-\mathrm{N}$ angle of $109.39^{\circ}$ (ideal $109.5^{\circ}$ ). The mean $\mathrm{Ag}-\mathrm{N}$ distance of $2.2845 \AA$ in $\left[\mathrm{Ag}(\mathrm{ACN})_{4}\right][\mathrm{FAP}]$ is slightly longer compared to the other complexes. However, no 
comparable Ag-F interactions were detected as the silver ion is completely shielded by the four coordinating acetonitrile ligands.

The $[\mathrm{FAP}]^{-}$anion crystallized in a distorted octahedral arrangement with the two axial perfluoroethyl groups turned slightly away from the third one. The axial angles were in the range between $168.6^{\circ}$ and $177.6^{\circ}$. Only the meridional conformation was formed for all three complexes, however, a disorder of the tris(perfluoroethyl)trifluorophosphate anions in the crystals of $\left[\mathrm{Ag}(\mathrm{ACN})_{4}\right][\mathrm{FAP}]$ and $\left[\mathrm{Ag}(\mathrm{ACN})_{2}\right][\mathrm{FAP}]$ with two orientations each was detected. The refined occupation probabilities for the two orientations were $53.6(1) \%$ and $46.4(1) \%$ for $\left[\mathrm{Ag}(\mathrm{ACN})_{2}\right][\mathrm{FAP}]$ and $53.5(2) \%$ and $46.5(2) \%$ for $\left[\mathrm{Ag}(\mathrm{ACN})_{4}\right][\mathrm{FAP}]$. These structures for the FAP anion coincide very well with earlier published crystal structures for $\mathrm{M}[\mathrm{FAP}]$ complexes. $^{36}$

\section{Physicochemical properties}

Liquefaction by propene absorption. When applying neat silver salt complexes as liquid absorption materials it is crucial for the separation performance to keep the absorption material always in the liquid state. Especially if the operation temperature lies below the melting point of the propene free silver salt, it is important to know the minimum partial pressure of propene necessary for the formation of the liquid silver-propene complex to avoid recrystallization. Therefore, we investigated the reaction of propene with the different $\mathrm{Ag}[\mathrm{FAP}]$ salts in more detail. As assumed before, all synthesized $\mathrm{Ag}[\mathrm{FAP}]$ complexes, except $\left[\mathrm{Ag}\left(\mathrm{C}_{3} \mathrm{H}_{6}\right)_{x}\right][\mathrm{FAP}]$, can indeed be liquefied at $30{ }^{\circ} \mathrm{C}$ when exposed to a certain propene pressure. The addition of a second ligand apparently leads to the disruption of the previous crystal structure and the formation of a new complex with very low lattice energy. Depending on the type and number of the ligands marked differences in the liquefaction pressures were observed, ranging from 0.01 bar for $\left[\mathrm{Ag}(\mathrm{ACN})_{2}\right][\mathrm{FAP}]$ to 9.38 bar for $[\mathrm{Ag}(\mathrm{en})][\mathrm{FAP}]$ (Table 2). Increasing the ligand number of same ligands (ACN: $2 \rightarrow 4$ ) results in an increase of the liquefaction pressure as easily accessible coordination sites at the silver ion are blocked by these additional ligands. As a general observation it can be stated that all nitrile-Ag[FAP] complexes with two ligands readily absorb propene already at low pressures. The elevated liquefaction pressures of the pyridine ( $5.75 \mathrm{bar}$ ) and ethylendiamine ( $9.38 \mathrm{bar}$ ) complexes have their origin in diverging structures in the solid state: the crystal structure of $\left[\mathrm{Ag}(\mathrm{py})_{2}\right][\mathrm{FAP}]$ reveals a dimeric arrangement of silver with interactions between adjacent pyridine rings impeding propene absorption. For $[\mathrm{Ag}(\mathrm{en})][\mathrm{FAP}]$ a polymeric

Table 2 Propene liquefaction pressures of the Ag[FAP] complexes at $30^{\circ} \mathrm{C}$

\begin{tabular}{ll} 
Silver salt & $\begin{array}{l}\text { Liquefaction pressure } \\
\text { at } 30{ }^{\circ} \mathrm{C} / \mathrm{bar}\end{array}$ \\
\hline$\left[\mathrm{Ag}(\mathrm{ACN})_{4}\right][\mathrm{FAP}]$ & $1.70 \pm 0.11$ \\
{$\left[\mathrm{Ag}(\mathrm{ACN})_{2}\right][\mathrm{FAP}]$} & 0.01 \\
{$\left[\mathrm{Ag}(\mathrm{Cl}-\mathrm{ACN})_{2}\right][\mathrm{FAP}]$} & $-{ }^{a}$ \\
{$\left[\mathrm{Ag}(\text { acryl-CN })_{2}\right][\mathrm{FAP}]$} & $0.10 \pm 0.05$ \\
{$\left[\mathrm{Ag}(\mathrm{py})_{2}\right][\mathrm{FAP}]$} & $5.75 \pm 0.05$ \\
{$[\mathrm{Ag}(\mathrm{en})][\mathrm{FAP}]$} & $9.38 \pm 0.05$ \\
{$\left[\mathrm{Ag}\left(\mathrm{C}_{3} \mathrm{H}_{6}\right)_{x}\right][\mathrm{FAP}]$} & $-{ }^{b}$ \\
${ }^{a} \mathrm{mp}<\mathrm{RT}^{b}{ }^{b}$ No liquefaction up to $10 \mathrm{bar}$.
\end{tabular}

structure similar to $[\mathrm{Ag}(\mathrm{en})]\left[\mathrm{NTf}_{2}\right]$ can be supposed. ${ }^{59}$ Removing propene from the previously liquefied silver FAP salts led either to spontaneous recrystallization (in case of $\left[\mathrm{Ag}(\mathrm{py})_{2}\right][\mathrm{FAP}]$, $[\mathrm{Ag}(\mathrm{en})][\mathrm{FAP}])$ or to a supercooled melt (in case of $\left[\mathrm{Ag}(\mathrm{ACN})_{2}\right]-$ [FAP], $\left.[\text { Ag(acryl-CN })_{2}\right][$ FAP $\left.]\right)$, from which crystals grew during a few days.

Density and thermal properties. For further characterization of the synthesized Ag[FAP] complexes, relevant physicochemical properties for their application in gas separation were determined (Table 3). Except for [Ag(en)][FAP], all synthesized silver FAP salts have a melting point below $100{ }^{\circ} \mathrm{C}$ with $\left[\mathrm{Ag}(\mathrm{Cl}-\mathrm{ACN})_{2}\right][\mathrm{FAP}]$ even being liquid at room temperature. These low melting points allow new application scenarios in comparison to other liquefiable but higher melting silver salts, such as $\mathrm{Ag}\left[\mathrm{NTf}_{2}\right] \cdot{ }^{29,30}$ Working above the melting point of the silver salts solves the problem of recrystallization in absence of propene pressure. This also enables the application of $\left[\mathrm{Ag}\left(\mathrm{C}_{3} \mathrm{H}_{6}\right)_{x}\right][\mathrm{FAP}]\left(\mathrm{mp}=53{ }^{\circ} \mathrm{C}\right)$ as liquid absorption material at elevated temperature although this salt could not be liquefied by increasing propene pressure at $30{ }^{\circ} \mathrm{C}$ as mentioned before.

The densities of the propene free ligand-Ag[FAP] salts range from $1.911 \mathrm{~g} \mathrm{~mL}^{-1}$ to $2.516 \mathrm{~g} \mathrm{~mL}^{-1}$ and increase with decreasing number of ligands. After exothermic propene coordination, a density drop to about $1.7 \mathrm{~g} \mathrm{~mL}^{-1}$ is determined for $\left[\mathrm{Ag}(\mathrm{ACN})_{2}\right][\mathrm{FAP}],\left[\mathrm{Ag}(\mathrm{Cl}-\mathrm{ACN})_{2}\right][\mathrm{FAP}]$ and $\left[\mathrm{Ag}(\text { acryl-CN })_{2}\right][\mathrm{FAP}]$ together with a strong volume expansion: for example, $10 \mathrm{~mL}$ of solid $\left[\mathrm{Ag}(\mathrm{ACN})_{2}\right][\mathrm{FAP}]$ turn into $13.84 \mathrm{~mL}$ of liquid $\left[\mathrm{Ag}(\mathrm{ACN})_{2}\left(\mathrm{C}_{3} \mathrm{H}_{6}\right)_{x}\right][\mathrm{FAP}]$ at $30{ }^{\circ} \mathrm{C}$ and 1 bar propene. Similar observations were also made for $\mathrm{Ag}\left[\mathrm{NTf}_{2}\right]$ during propene absorption. ${ }^{29}$

In addition, TGA measurements with a heating rate of $5 \mathrm{~K}$ $\min ^{-1}$ provided information on the thermal stability of the propene free silver salts and the reversibility of the propene absorption at the silver FAP complexes under investigation (Table 3). The temperature $T_{0.2 \mathrm{~m} \%}$, at which $0.2 \%$ of the original mass is lost, is taken as the limit of incipient decomposition by ligand abstraction. $T_{\text {onset }}$, in contrast, indicates strong mass loss on further heating, for example by total decomposition of the anion. The TGA results show a starting decomposition of $\left[\mathrm{Ag}(\mathrm{ACN})_{4}\right][\mathrm{FAP}]$ and $\left[\mathrm{Ag}(\mathrm{Cl}-\mathrm{ACN})_{2}\right][\mathrm{FAP}]$ already at temperatures

Table 3 Density, melting point and thermal stability of the synthesized Ag[FAP] salts

\begin{tabular}{lllll}
\hline & $\begin{array}{l}\text { Density } / \mathrm{g} \mathrm{mL}^{-1} \\
\left(20{ }^{\circ} \mathrm{C}\right)\end{array}$ & $\mathrm{mp} /{ }^{\circ} \mathrm{C}$ & \multicolumn{2}{c}{ Therm. stability $/{ }^{\circ} \mathrm{C}$} \\
\cline { 5 - 6 } Silver salt & 1.911 & 44 & 40 & 54,213 \\
\hline$\left[\mathrm{Ag}(\mathrm{ACN})_{4}\right][\mathrm{FAP}]$ & 2.140 & 31 & 127 & 212 \\
{$\left[\mathrm{Ag}(\mathrm{ACN})_{2}\right][\mathrm{FAP}]$} & 2.034 & $<\mathrm{rt}^{a}$ & 81 & 182 \\
{$\left[\mathrm{Ag}(\mathrm{Cl}-\mathrm{ACN})_{2}\right][\mathrm{FAP}]$} & 44 & 122 & 155,212 \\
{$\left[\mathrm{Ag}(\text { acryl-CN })_{2}\right][\mathrm{FAP}]$} & 2.063 & 81 & 146 & 271 \\
{$\left[\mathrm{Ag}(\mathrm{py})_{2}\right][\mathrm{FAP}]$} & 2.035 & 141 & 175 & 218 \\
{$[\mathrm{Ag}(\mathrm{en})][\mathrm{FAP}]$} & 2.516 & 53 & 30 & 30,149 \\
{$\left[\mathrm{Ag}\left(\mathrm{C}_{3} \mathrm{H}_{6}\right)_{x}\right][\mathrm{FAP}]$} & $-{ }^{b}$ & &
\end{tabular}

${ }^{a}$ None detectable in the DSC experiment down to $-50{ }^{\circ} \mathrm{C}$, yet crystallization was observed in the freezer at $-18{ }^{\circ} \mathrm{C}$ after one week. ${ }^{b}$ Not accessible by normal methods due to loss of propene; uncertainties are up to $\pm 0.015 \mathrm{~g} \mathrm{~mL}^{-1}$ in the density measurements, up to $\pm 3{ }^{\circ} \mathrm{C}$ in the melting point determination, and up to $\pm 8{ }^{\circ} \mathrm{C}$ in the thermal stability determinations. 
below $100{ }^{\circ} \mathrm{C}$. These findings can be explained by the weakened metal-ligand bond with increasing number of ligands and by the presence of the electron withdrawing chlorine atom in the ligand.

In general, moderate temperatures should be applied for all $\mathrm{N}$-coordinated Ag-complexes to avoid loss of ligands. Our experimental observations hint to the fact that a few strongly binding ligands are more favorable for thermal stability compared to a high number of weakly coordinating ligands. Moreover, the TGA measurements of the propene silver FAP complexes indicate a good reversibility of propene absorption with beginning desorption already at $30{ }^{\circ} \mathrm{C}$ for all synthesized complexes.

\section{Theoretical propene/propane separation performance}

Propene uptake. A very important performance indicator for the applicability of the developed $\mathrm{Ag}[\mathrm{FAP}]$ salts in propene/ propane separation is propene uptake and maximum propene capacity. Therefore, pressure dependent single gas measurements of propene solubility in the synthesized silver FAP salts were carried out in the isochoric gas solubility cell shown in Fig. 1. Silver utilization, gravimetric and volumetric capacity at $30{ }^{\circ} \mathrm{C}$ are depicted in Fig. 4. Chemical absorption and formation of the propene-silver complex salts is characterized by the steep increase in the propene up-take at low propene pressure. The following linear part at higher propene pressures represents the physical absorption of propene in the formed liquid Ag-propene complex salt.

These characteristic pressures in the propene uptake curves match very well with the previously determined liquefaction pressures for the different complexes. The Ag[FAP] complexes with two nitrile ligands exhibit the highest propene uptake with small differences probably resulting from the different coordination strength and polarity of the ligands. Ag[FAP] salts with four ACNs, py and en as ligands show, in contrast, significantly lower propene uptake due to competition for coordination sites. For previously evacuated $\left[\mathrm{Ag}\left(\mathrm{C}_{3} \mathrm{H}_{6}\right)_{x}\right][\mathrm{FAP}]$, a maximum propene uptake of $1 \mathrm{~mol}$ propene per mol of silver was determined already at low pressures. Compared to solutions of silver salts in ionic liquids, ${ }^{11-13}$ similar silver utilizations are achieved, but due to a higher Ag concentration without the additional solvent the gravimetric and volumetric capacities of the here presented systems are by a factor of 2-4 higher. However, due to the relatively high molecular weight of the $[\mathrm{FAP}]^{-}$ion and the partly ligand blocked coordination sites, the extremely high capacity of neat $\mathrm{Ag}\left[\mathrm{NTf}_{2}\right]^{29}$ is not reached with the here described $\mathrm{Ag}[\mathrm{FAP}]$ systems.

Mixed gas solubility and selectivity of solubility. Previous investigations of our group revealed a strong influence of propene solubility on propane solubility for the liquid silver complex $\left[\operatorname{Ag}\left(\mathrm{C}_{3} \mathrm{H}_{6}\right)_{x}\right]\left[\mathrm{NTf}_{2}\right]^{29}{ }^{29}$ Therefore, we decided to perform mixed gas measurements in order to allow a more realistic prediction of the membrane performance and equilibrium absorption selectivity. Pressure NMR proved to be a powerful tool to determine solubilities, diffusion coefficients and selectivities with only one sample and two measurements (qNMR + PFG-NMR). The target values were obtained at a total pressure of 6 bar with a 50/50 mol\% mixture of propene and propane for a detailed
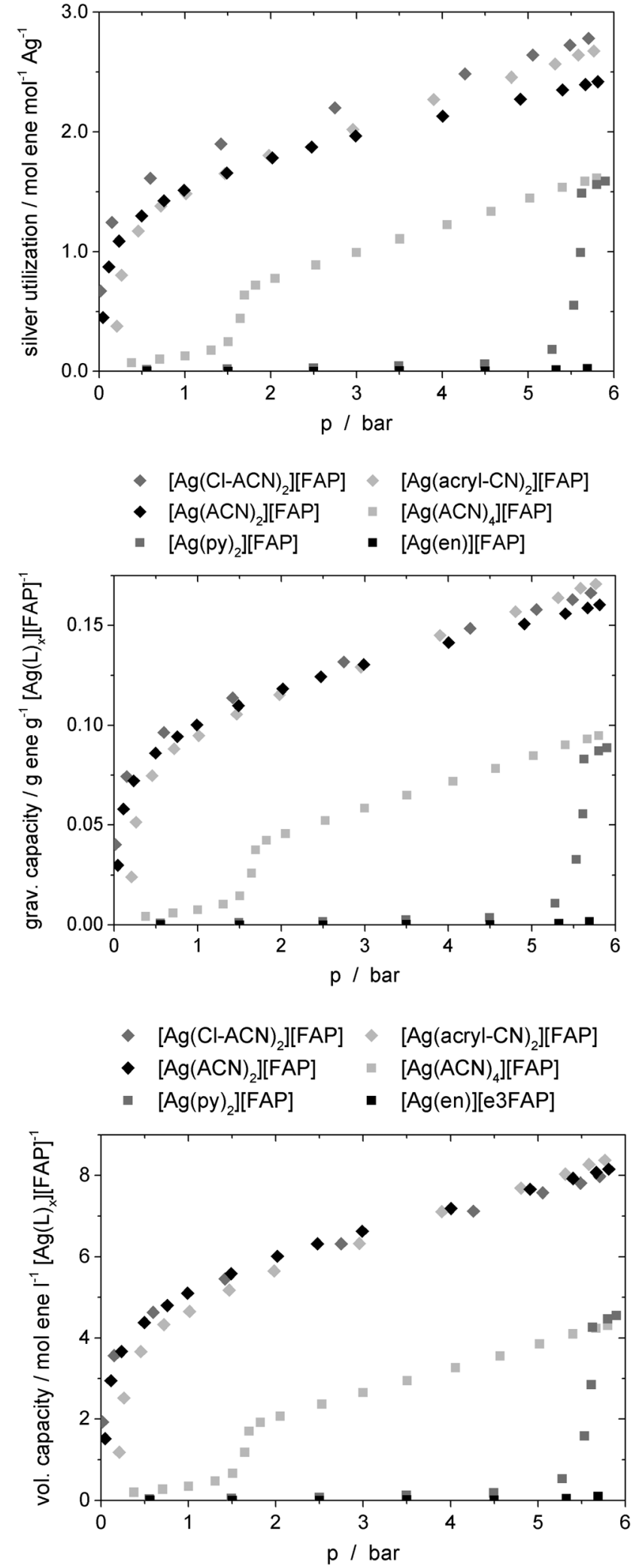

Fig. 4 Pressure dependent propene uptake of the investigated Ag[FAP] complexes determined by single gas measurements in an isochoric gas solubility cell at $30{ }^{\circ} \mathrm{C}$. Top: Silver utilization. Middle: Gravimetric capacity. Bottom: Volumetric capacity. 
comparison of the Ag[FAP] complexes both at 30 and $70{ }^{\circ} \mathrm{C}$. Due to a missing additional ligand as internal standard, the determination of absolute values for the solubility of propene and propane in $\left[\mathrm{Ag}\left(\mathrm{C}_{3} \mathrm{H}_{6}\right)_{x}\right][\mathrm{FAP}]$ was not possible. $\left[\mathrm{Ag}(\mathrm{py})_{2}\right][\mathrm{FAP}]$ and $[\mathrm{Ag}(\mathrm{en})][\mathrm{FAP}]$ were not investigated further due to their high liquefaction pressures. The determined absorption equilibrium selectivity or selectivity of solubility for propene is defined as the ratio of the solubility of propene and propane referenced to their individual partial pressures:

$$
S_{\mathrm{sol}}^{\mathrm{ene}}=\frac{c_{\text {ene }} \times p_{\text {ane }}}{c_{\text {ane }} \times p_{\text {ene }}} .
$$

The mixed gas solubilities of propene and propane in the studied Ag[FAP] salts are shown in Fig. 5. Propane solubilities are low compared to propene solubilities as the paraffin is only physically absorbed in the liquid salts. This results in generally good selectivities of solubility for propene (Fig. 7).

A detailed comparison of the silver salts shows that, except for $\left[\mathrm{Ag}(\mathrm{ACN})_{4}\right][\mathrm{FAP}]$, only minor differences in the solubility and selectivity are detected for the nitrile ligated complexes. On temperature increase from 30 to $70{ }^{\circ} \mathrm{C}$, both propane and propene solubility decrease. However, the relative decrease of propene is much smaller due to its stronger binding by chemical complexation. This results in 2 to 3 times higher selectivities of solubility at $70{ }^{\circ} \mathrm{C}$. Through its coordination by four acetonitrile ligands, $\left[\mathrm{Ag}(\mathrm{ACN})_{4}\right][\mathrm{FAP}]$ exhibits a lower solubility and selectivity for propene, while the propane solubility lies in the same range as for the other nitrile complexes. Moreover, only a very small selectivity improvement is observed by rising temperature, due to the weaker propene silver interactions. The negative influence of an increasing ligand number is due to the blocking of coordination sites for propene. In contrast, $\left[\mathrm{Ag}\left(\mathrm{C}_{3} \mathrm{H}_{6}\right)_{x}\right][\mathrm{FAP}]$ (with no competing ligands) exhibits at least twice the equilibrium absorption selectivity $\left(S_{\text {sol }}=39\right)$ compared to the other Ag[FAP] salts. This is an even higher selectivity of solubility than found for $\left[\operatorname{Ag}\left(\mathrm{C}_{3} \mathrm{H}_{6}\right)_{x}\right]\left[\mathrm{NTf}_{2}\right]$ $\left(S_{\text {sol }}=33\right)$ under otherwise identical conditions.

Diffusion and membrane selectivity. The solution diffusion model, which describes the transport through dense membranes,

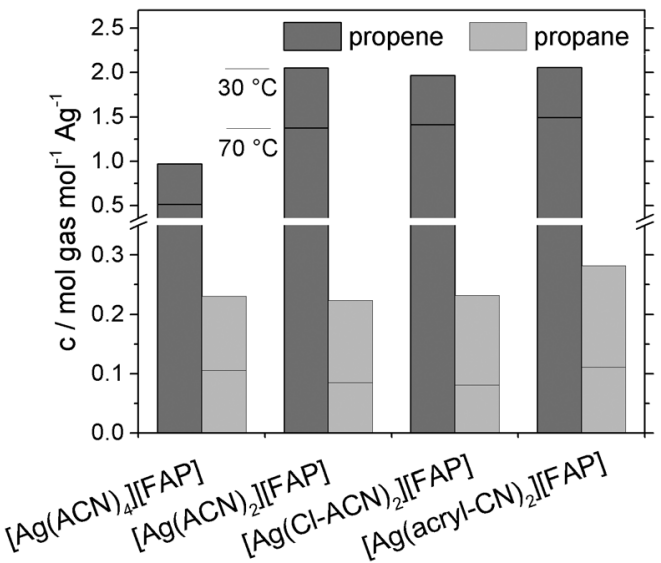

Fig. 5 Propene and propane solubility in the different silver FAP complexes at $30{ }^{\circ} \mathrm{C}$ and $70{ }^{\circ} \mathrm{C}$ and 3 bar propene/3 bar propane pressure obtained by mixed gas qNMR measurements. allows for a theoretical prediction of the separation performance of the $\mathrm{Ag}[\mathrm{FAP}]$ complexes as liquid membrane material. According to the solution diffusion model, the membrane selectivity can be calculated from the ratio of the solubility selectivity and the diffusion coefficients of propene and propane as follows:

$$
S_{\mathrm{mem}}^{\mathrm{ene}}=S_{\mathrm{sol}}^{\mathrm{ene}} \times \frac{D_{\text {ene }}}{D_{\mathrm{ane}}} .
$$

Self diffusion coefficients of propene and propane in the liquid salts were determined by PFG-NMR and inserted into the above equation to predict the membrane selectivity as described in a previous publication of some of us. ${ }^{29}$ Although the diffusion coefficients of molecules of very similar size, such as propene and propane, should be very similar, distinctive differences are observed in all investigated silver salts (Fig. 6). These differences originate from the fact that most of the propene is bound to silverions in form of comparatively large and thus slower diffusing complexes. This leads to a measured, average diffusion coefficient of propene that is always much smaller than that of propane. Consequently, the positive influence of chemical complexation on the solubility of propene is partly corrupted by its negative influence on diffusion. Still, the overall membrane selectivity is in favor of propene for all $\mathrm{Ag}[\mathrm{FAP}]$ salts (Fig. 7) revealing interesting overall membrane selectivity values. All these observations made for the $\mathrm{Ag}[\mathrm{FAP}]$ complexes coincide well with those made earlier for $\left[\mathrm{Ag}\left(\mathrm{C}_{3} \mathrm{H}_{6}\right)_{x}\right]\left[\mathrm{NTf}_{2}\right]^{29}$

Despite the fact that the ratio of the diffusion coefficients of propene and propane is always smaller than one, prominent differences are found for the differently ligated $\mathrm{Ag}[\mathrm{FAP}] \mathrm{s}$. Diffusion coefficients for propene increase from $\left[\mathrm{Ag}\left(\mathrm{C}_{3} \mathrm{H}_{6}\right)_{x}\right][\mathrm{FAP}]$ over $\left[\mathrm{Ag}(\mathrm{Cl}-\mathrm{ACN})_{2}\right][\mathrm{FAP}],\left[\mathrm{Ag}(\mathrm{ACN})_{2}\right][\mathrm{FAP}]$ and $\left[\mathrm{Ag}(\text { acryl-CN })_{2}\right][\mathrm{FAP}]$ to $\left[\mathrm{Ag}(\mathrm{ACN})_{4}\right][\mathrm{FAP}]$. Decreasing coordination strength of propene and decreasing viscosity of the liquid salts according to the StokesEinstein equation explain this sequence. Increasing temperature leads to increasing diffusion coefficients of propene and propane, as expected. However, due to the complexation of propene, the relative increase of the Ag-complex diffusion coefficients is always

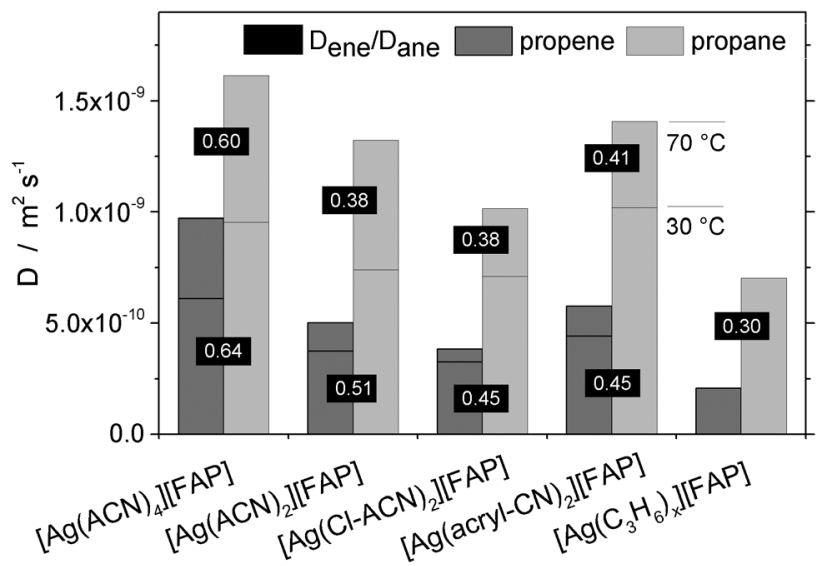

Fig. 6 Diffusion coefficients of propene and propane in $\left[\mathrm{Ag}(\mathrm{ACN})_{4}\right][\mathrm{FAP}]$, $\left[\mathrm{Ag}(\mathrm{ACN})_{2}\right][\mathrm{FAP}],\left[\mathrm{Ag}(\mathrm{Cl}-\mathrm{ACN})_{2}\right][\mathrm{FAP}],\left[\mathrm{Ag}(\mathrm{acryl}-\mathrm{CN})_{2}\right][\mathrm{FAP}]$ and $\left[\mathrm{Ag}\left(\mathrm{C}_{3} \mathrm{H}_{6}\right)_{x}\right][\mathrm{FAP}]$ at $30{ }^{\circ} \mathrm{C}$ and $70{ }^{\circ} \mathrm{C}$ and 3 bar propene/3 bar propane pressure obtained by mixed gas PFG-NMR measurements. 


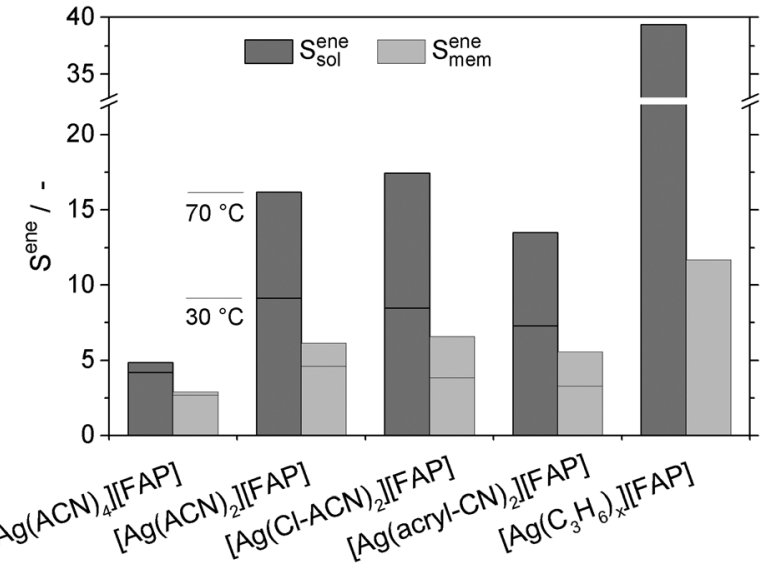

Fig. 7 Selectivity of solubility and membrane selectivity of propene in the propene/propane separation using Ag[FAP] salts at $30{ }^{\circ} \mathrm{C}$ and $70{ }^{\circ} \mathrm{C}$ and 3 bar propene $/ 3$ bar propane pressure obtained by mixed gas NMR measurements.

smaller from 30 to $70{ }^{\circ} \mathrm{C}$ in comparison to propane. Interestingly, with the exception of $\left[\mathrm{Ag}\left(\mathrm{C}_{3} \mathrm{H}_{6}\right)_{x}\right][\mathrm{FAP}]$, diffusion of propene and propane in all $\mathrm{Ag}[\mathrm{FAP}]$ complexes is faster than in $\left[\mathrm{Ag}\left(\mathrm{C}_{3} \mathrm{H}_{6}\right)_{x}\right]\left[\mathrm{NTf}_{2}\right]$ $\left(D_{\text {ene }}=2.39 \times 10^{-10} \mathrm{~m}^{2} \mathrm{~s}^{-1}, D_{\text {ane }}=5.86 \times 10^{-10} \mathrm{~m}^{2} \mathrm{~s}^{-1}\right.$ at $\left.70{ }^{\circ} \mathrm{C}\right)$. This faster diffusion leads to a higher flux and desirable faster separation in liquid membrane based on $\mathrm{Ag}[\mathrm{FAP}]$ salts compared to $\mathrm{Ag}\left[\mathrm{NTf}_{2}\right]$ salts.

\section{Conclusions}

Our investigations show that liquid silver salts formed by alkene complexation of $\mathrm{Ag}[\mathrm{FAP}]$ salts are promising liquid absorption materials for alkene/alkane separation, such as liquid membrane or absorption/desorption processes. The weakly coordinating FAP anion requires additional ligands to form stable alkene complexes. The choice and variability of these ligands enable additional options to tune the physicochemical properties of these novel liquid absorption agents for optimized separation processes.

A newly developed synthesis route for $\mathrm{Ag}[\mathrm{FAP}]$ salts is also presented. The latter uses $\mathrm{K}[\mathrm{FAP}]$ and $\mathrm{Ag}\left[\mathrm{NO}_{3}\right]$ and allows, in contrast to literature known protocols, ${ }^{36}$ the use of cheap and easy-to-handle reagents for a straight forward preparation of a wide variety of different liquid $\left[\mathrm{Ag}(\text { ligand })_{x}\right][\mathrm{FAP}]$ salts. Crystal structures of selected silver complexes confirm that the choice of ligand has a strong influence on the crystal structure and physicochemical properties of the new Ag[FAP] salts.

Compared to the previously published $\mathrm{Ag}\left[\mathrm{NTf}_{2}\right]$-alkene salts, ${ }^{29}$ lower liquefaction pressures and much lower melting points of the $\mathrm{Ag}[\mathrm{FAP}]$ salts before propene absorption have been found. This may exclude the risk of recrystallization when performing ene/ane-separation processes above the salt's melting point. To guarantee the stability of the Ag[FAP] complexes, moderate temperatures below $100{ }^{\circ} \mathrm{C}$ should be applied in applications as loss of ligands and complex decomposition has been shown to set in at relatively low temperatures.
The investigation of the separation performance revealed that, although the additional ligands at the silver ion are blocking coordination sites for propene, the right number and type of ligands still enables high capacities. It has been found that a small number of strongly coordinating ligands forming $\left[\mathrm{Ag}(\text { ligand })_{x}\right][\mathrm{FAP}]$ complexes with low lattice energies offer the most interesting systems for propene/propane separation processes.

While selective chemical complexation of propene to silver leads to high selectivity of solubility, slow diffusion of the silver-propene complex reduces membrane selectivity. Still high membrane selectivities of up to 12 are predicted from the performed solubility and diffusion measurements. Compared to $\mathrm{Ag}\left[\mathrm{NTf}_{2}\right]$-alkene salts, the analogues $[\mathrm{FAP}]^{-}$-salts reported here show higher diffusivity and thus promise higher membrane fluxes.

\section{Acknowledgements}

The authors would like to acknowledge the funding of the Deutsche Forschungsgemeinschaft (DFG) and Merck KGaA through the Cluster of Excellence Engineering of Advanced Materials. The authors would also like to thank Dr Nicola Taccardi for performing the ICP-AES measurements.

\section{References}

1 Ceresana, Market Study: Propylene, Ceresana, Konstanz, 2014.

2 J. H. Kim, B. R. Min, J. Won, S. H. Joo, H. S. Kim and Y. S. Kang, Macromolecules, 2003, 36, 6183-6188.

3 ICIS, Propylene Uses and Market Data, http://www.icis.com/ resources/news/2007/11/06/9076455/propylene-uses-andmarket-data/, accessed 11.08.2016, 2016.

4 P. F. Bryan, Sep. Purif. Rev., 2004, 33, 157-182.

5 R. B. Eldrige, Ind. Eng. Chem. Res., 1993, 32, 2208-2212.

6 B. B. Baker, Inorg. Chem., 1964, 3, 200-202.

7 I. H. Cho, H. K. Yasuda and T. R. Marrero, J. Chem. Eng. Data, 1995, 40, 107-111.

8 H. L. Clever, E. R. Baker and W. R. Hale, J. Chem. Eng. Data, 1970, 15, 411-413.

9 W. Featherstone and A. J. S. Sorrie, J. Chem. Soc., 1964, 5235-5242, DOI: 10.1039/jr9640005235.

10 A. E. M. George, E. Keller, S. K. Verma and K. D. Williamson, in Membrane and Purification Technology, ed. J. M. C. Norman and N. Li, Marcel Dekker, Inc., New York, 1992, ch. 3, pp. 59-83.

11 M. Fallanza, A. Ortiz, D. Gorri and I. Ortiz, J. Chem. Eng. Data, 2013, 58, 2147-2153.

12 S. L. M. Galan, G. W. Meindersma and A. B. Haan, Ind. Eng. Chem. Res., 2009, 48, 10650-10656.

13 A. Ortiz, L. M. Galan, D. Gorri, A. B. de Haan and I. Ortiz, Ind. Eng. Chem. Res., 2010, 49, 7227-7233.

14 A. Ortiz, A. Ruiz, D. Gorri and I. Ortiz, Sep. Purif. Technol., 2008, 63, 311-318. 
15 H. W. Krekeler, J. M. Hirschbeck and U. Schwenk, Erdoel Kohle, 1963, 16, 551-560.

16 H. W. Quinn, Prog. Sep. Purif., 1971, 4, 133-169.

17 A. Ortiz, L. M. Galan, D. Gorri, A. B. de Haan and I. Ortiz, Sep. Purif. Technol., 2010, 73, 106-113.

18 N. R. Brooks, S. Schaltin, K. Van Hecke, L. Van Meervelt, J. Fransaer and K. Binnemans, Dalton Trans., 2012, 41, 6902-6905.

19 S. Schaltin, N. R. Brooks, J. Sniekers, D. Depuydt, L. Van Meervelt, K. Binnemans and J. Fransaer, Phys. Chem. Chem. Phys., 2013, 15, 18934-18943.

20 S. Schaltin, N. R. Brooks, L. Stappers, K. Van Hecke, L. Van Meervelt, K. Binnemans and J. Fransaer, Phys. Chem. Chem. Phys., 2012, 14, 1706-1715.

21 J. Sniekers, N. R. Brooks, S. Schaltin, L. Van Meervelt, J. Fransaer and K. Binnemans, Dalton Trans., 2014, 43, 1589-1598.

22 T. Vander Hoogerstraete, N. R. Brooks, B. Norberg, J. Wouters, K. Van Hecke, L. Van Meervelt and K. Binnemans, CrystEngComm, 2012, 14, 4902-4911.

23 J.-F. Huang, H. Luo, C. Liang, D.-e. Jiang and S. Dai, Ind. Eng. Chem. Res., 2008, 47, 881-888.

24 Y. Wang, W. Hao, J. Jacquemin, P. Goodrich, M. Atilhan, M. Khraisheh, D. Rooney and J. Thompson, J. Chem. Eng. Data, 2015, 60, 28-36.

25 Y. Wang, J. Thompson, J. Zhou, P. Goodrich, M. Atilhan, A. S. Pensado, B. Kirchner, D. Rooney, J. Jacquemin and M. Khraisheh, J. Chem. Thermodyn., 2014, 77, 230-240.

26 A. W. Francis, J. Am. Chem. Soc., 1951, 73, 3709-3713.

27 H. W. Quinn and D. N. Glow, Can. J. Chem., 1962, 40, 1103-1112.

28 H. W. Quinn, J. S. McIntyre and D. J. Peterson, Can. J. Chem., 1965, 43, 2896-2910.

29 F. Agel, F. Pitsch, F. F. Krull, P. Schulz, M. Wessling, T. Melin and P. Wasserscheid, Phys. Chem. Chem. Phys., 2011, 13, 725-731.

30 F. Pitsch, F. F. Krull, F. Agel, P. Schulz, P. Wasserscheid, T. Melin and M. Wessling, Adv. Mater., 2012, 24, 4306-4310.

31 M. Stricker, B. Oelkers, C. P. Rosenau and J. Sundermeyer, Chem. - Eur. J., 2013, 19, 1042-1057.

32 N. V. Ignat'ev, U. Welz-Biermann, A. Kucheryna, G. Bissky and H. Willner, J. Fluorine Chem., 2005, 126, 1150-1159.

33 N. V. Ignat'ev, H. Willner and P. Sartori, J. Fluorine Chem., 2009, 130, 1183-1191.

34 M. Schmidt, U. Heider, A. Kuehner, R. Oesten, M. Jungnitz, N. Ignat'ev and P. Sartori, J. Power Sources, 2001, 97-98, 557-560.
35 C. Yao, W. R. Pitner and J. L. Anderson, Anal. Chem., 2009, 81, 5054-5063.

36 C. Nikolaou, PhD thesis, Heinrich-Heine-Universität Düsseldorf, 2009.

37 SADABS 2014/4, Bruker AXS, Inc., Madison, WI, USA, 2014.

38 SADABS 2012/1, Bruker AXS, Inc., Madison, WI, USA, 2012.

39 SHELXTL NT 6.12, Bruker AXS, Inc., Madison, WI, USA, 2002.

40 G. M. Sheldrick, Acta Crystallogr., Sect. C: Struct. Chem., 2015, 71, 3-8.

41 C. S. Johnson, Jr., Prog. Nucl. Magn. Reson. Spectrosc., 1999, 34, 203-256.

42 J. Kaerger, in Adsorption and Diffusion, ed. J. W. Hellmut and G. Karge, Springer, Berlin Heidelberg, 2008, vol. 7, pp. 85-133.

43 J. Kaerger, H. Pfeifer and W. Heink, Adv. Magn. Reson., 1988, 12, 1-89.

44 W. S. Price, Concepts Magn. Reson., 1997, 9, 299-336.

45 W. S. Price, Concepts Magn. Reson., 1998, 10, 197-237.

46 P. Stilbs, Prog. Nucl. Magn. Reson. Spectrosc., 1987, 19, 1-45.

47 D. Sinnaeve, Concepts Magn. Reson., Part A, 2012, 40, 39-65.

48 D. Wu, A. Chen and C. S. Johnson, Jr., J. Magn. Reson., Ser. A, 1995, 115, 260-264.

49 E. O. Stejskal and J. E. Tanner, J. Chem. Phys., 1965, 42, 288-292.

50 M. Holz, S. R. Heil and A. Sacco, Phys. Chem. Chem. Phys., 2000, 2, 4740-4742.

51 N. Ignatyev, M. Schmidt, A. Kühner, V. Hilarius, U. Heider, A. Kucheryna, P. Sartori and H. Willner, WO2003002579A1, Merck Patent GmbH, 2003.

52 J.-F. Huang, H. Luo and S. Dai, J. Electrochem. Soc., 2006, 153, J9-J13.

53 A. A. M. Aly, B. Walfort and H. Lang, Z. Kristallogr. - New Cryst. Struct., 2004, 219, 489-491.

54 M. Vierle, Y. Zhang, A. M. Santos, K. Koehler, C. Haessner, E. Herdtweck, M. Bohnenpoll, O. Nuyken and F. E. Kuehn, Chem. - Eur. J., 2004, 10, 6323-6332.

55 Y. Zhang, A. M. Santos, E. Herdtweck, J. Mink and F. E. Kuehn, New J. Chem., 2005, 29, 366-370.

56 C. Janiak, Dalton Trans., 2000, 3885-3896.

57 S. Alvarez, Dalton Trans., 2013, 42, 8617-8636.

58 T. G. Gray and J. P. Sadighi, in Molecular Metal-Metal Bonds: Compounds, Synthesis, Properties, ed. S. T. Liddle, Wiley-VCH, Weinheim, 1st edn, 2015, pp. 403-406.

59 D. Depuydt, N. R. Brooks, S. Schaltin, L. Van Meervelt, J. Fransaer and K. Binnemans, ChemPlusChem, 2013, 78, 578-588. 